\title{
Random Evolution Equations: Well-Posedness, Asymptotics, and Applications to Graphs
}

\author{
Stefano Bonaccorsi ${ }^{1}$ (D) $\cdot$ Francesca Cottini ${ }^{2} \cdot$ Delio Mugnolo $^{3}$
}

Accepted: 11 November 2020 / Published online: 11 March 2021

(C) The Author(s) 2021

\begin{abstract}
We study diffusion-type equations supported on structures that are randomly varying in time. After settling the issue of well-posedness, we focus on the asymptotic behavior of solutions: our main result gives sufficient conditions for pathwise convergence in norm of the (random) propagator towards a (deterministic) steady state. We apply our findings in two environments with randomly evolving features: ensembles of difference operators on combinatorial graphs, or else of differential operators on metric graphs.
\end{abstract}

Keywords Operator semigroups · Evolution equations in random environments · Discrete Laplacians · Quantum graphs

Mathematics Subject Classification Primary 35R60 - Secondary 47D06, 37A50 , $60 \mathrm{~K} 15$

Dedicated to Professor Rainer Nagel on the occasion of his 80th birthday.

The third author was partially supported by the Deutsche Forschungsgemeinschaft (Grant 397230547).

$凶 \quad$ Stefano Bonaccorsi

stefano.bonaccorsi@unitn.it

Francesca Cottini

f.cottini2@campus.unimib.it

Delio Mugnolo

delio.mugnolo@fernuni-hagen.de

1 Dipartimento di Matematica, Università di Trento, via Sommarive 14, Povo, TN 38123, Italy

2 Dipartimento di Matematica e Applicazioni, Università di Milano Bicocca, via Roberto Cozzi 55, 20125 Milan, Italy

3 Lehrgebiet Analysis, Fakultät Mathematik und Informatik, FernUniversität in Hagen, 58084 Hagen, Germany 


\section{Introduction}

Randomly switching dynamical systems stand in between deterministic evolution equations (where the dynamics of the system is prescribed and completely known a priori) and stochastic differential equations, where the dynamics is perturbed by the introduction of noise.

Such systems are described by a continuous component, which follows a (deterministic) evolution driven by an operator $A_{j}$ which is selected among a class of operators $\mathrm{C}=\left\{A_{1}, \ldots, A_{n}\right\}$ by a discrete jump process.

These problems are related to a large-but somehow disjoint-literature, which treats piecewise deterministic Markov processes [5,11,12,29], switched dynamical systems [7], products of random matrices [22], random walk in random environment $[39,40]$ with applications in biology [10], physics [9] or finance [38], for instance.

In the present paper, we study the asymptotic behavior of a class of random evolution problems that may be relevant in some applications. Our main results (Theorems 2.11 and 2.13 below) state that the system consisting of a random switching between parabolic evolution equations driven by contractive, compact analytic semigroups satisfying suitable additional conditions converges towards an orthogonal projector provided the process spends enough time at each state, and we are able to estimate the rate of convergence: we refer to Sect. 2 for the theorems' formulation and Sect. 3 for their proof. As a motivation to our study, we provide in this section an example concerning the dynamics of the discrete heat equation on a system of random varying graphs. This example will be further analyzed in Sect. 4, which is devoted to the study of combinatorial graphs: there we discuss some further examples which relate our results to the existing literature. Finally, Sect. 5 is devoted to an application of our theory to a randomly switching evolution system on metric graphs. This section takes advantage of a novel formal definition of metric graphs [30] which can be exploited to verify the assumptions of our construction.

\subsection{A Motivating Example}

Let $G_{1}, \ldots, G_{N}$ be a family of simple (i.e., with no loops or multiple edges) but not necessarily connected graphs on a fixed set of vertices $V$ with cardinality $|\mathrm{V}|$. We consider the function space defined as the complex, finite-dimensional Hilbert space $\mathbb{C}^{\mathrm{V}} \equiv\{u: \mathrm{V} \rightarrow \mathbb{C}\}$.

On every graph $\mathrm{G}_{k}$ we introduce the graph Laplacian $\mathcal{L}_{k}$ (for a formal definition, see Sect. 4), which (under our convention on the sign) is negative semi-definite and whose eigenvalue $\lambda_{1}=0$ has multiplicity equal to the number of connected components in $\mathrm{G}_{k}$. The corresponding eigenspace is spanned by the collection of indicator functions on each connected component. In particular, if $G_{k}$ is connected, then $\operatorname{ker} \mathcal{L}_{k}=\langle\mathbb{1}\rangle$ is the space of constant functions on the vertices.

It is known ([31, Ch. 4]) that the solution of the Cauchy problem 


$$
\left\{\begin{aligned}
\frac{d}{d t} u(t, \mathrm{v}) & =\mathcal{L}_{k} u(t, \mathrm{v}), & & \mathrm{v} \in \mathrm{V}, t \geq 0, \\
u(0, \mathrm{v}) & =f(\mathrm{v}), & \mathrm{v} & \in \mathrm{V},
\end{aligned}\right.
$$

can be expressed in the form

$$
u(t, \mathrm{v})=e^{t \mathcal{L}_{k}} f(\mathrm{v})
$$

and in the limit for $t \rightarrow \infty$, it converges to the projector $P_{k} f$ onto the null space ker $\mathcal{L}_{k}$, where the projection equals the average of $f$ on each connected component of $\mathrm{G}_{k}$.

Let us introduce a random mechanism of switching the graphs over time. Having fixed a probability space $(\Omega, \mathcal{F}, \mathbb{P})$, we introduce a Markov chain $\left.\left\{X_{k}, k \geq 0\right\}\right)$ with state space $E=\{1, \ldots, N\}$ (which defines the environment where the evolution takes place) and a sequence of increasing random times $\left\{T_{k}, k \geq 0\right\}, T_{0}=0$, such that the Cauchy problem is defined by the operator $\mathcal{L}_{X_{k}}$ on the time interval $\left[T_{k}, T_{k+1}\right)$

$$
\left\{\begin{aligned}
\frac{d}{d t} u(t, \mathrm{v}) & =\mathcal{L}_{X_{k}} u(t, \mathrm{v}), & & \mathrm{v} \\
u(0, \mathrm{v}) & =f(\mathrm{v}), t \in\left[T_{k}, T_{k+1}\right), k \in \mathbb{N}, & & \mathrm{v} \in \mathrm{V} .
\end{aligned}\right.
$$

We can associate with (1.1) the random propagator

$$
S(t)=e^{\left(t-T_{n}\right) \mathcal{L}_{X_{n}}} \prod_{k=0}^{n-1} e^{\left(T_{k+1}-T_{k}\right) \mathcal{L}_{X_{k}}}, \quad t \in\left[T_{n}, T_{n+1}\right), n \in \mathbb{N},
$$

which maps each initial data $f \in \mathbb{C}^{\mathrm{V}}$ into the solution $u(t)$ of (1.1) at time $t$. This settles the issue of well-posedness of (1.1). The main question we are going to address in this paper is however the following:

(P) Does the random propagator $(S(t))_{t \geq 0}$ converge as $t \rightarrow \infty$ ? Towards which limit?

The asymptotic behavior of a random propagator $(S(t))_{t \geq 0}$ associated with problem (1.1) has not yet been studied in a general, possibly infinite-dimensional setting. Some results are known for finite-dimensional time-discrete dynamical systems, where the random propagator $\left(S\left(T_{n}\right)\right)_{n \in \mathbb{N}}$ defined likewise is a product of random matrices (PRM for short): this theory dates back to the 1960s, see e.g. Furstenberg [20].

In Theorem 2.11 we show that under suitable assumptions on the random switching mechanism and on the involved operators, the random propagator converges towards the orthogonal projection on $\bigcap_{j=1}^{N} \operatorname{ker} \mathcal{L}_{j}$; and in Theorem 2.13 we estimate the rate of convergence. In Sect. 2 we set the mathematical stage and then formulate both results; their proofs are led in Sect. 3, after collecting some necessary lemmata of probabilistic and operator theoretical nature. Our main results require an accurate analysis of the null spaces of the operators driving the relevant evolution equations (in 
our motivating example, the discrete Laplacians $\mathcal{L}_{k}$ ): notice that even when all $\operatorname{ker} \mathcal{L}_{k}$ have the same dimension, there is no reason why this should agree with the dimension of $K:=\bigcap_{j=1}^{N} \operatorname{ker} \mathcal{L}_{j}$; describing the orthogonal projector onto $K$ is therefore, not an easy task. Coming back to our motivating example of graphs, we observe in Sect. 4 that $K$ can be explicitly described in terms of the null space of a new operator $A$ that is related to the Laplacians on the graphs $G_{1}, \ldots, G_{N}$ but acts on a different class of functions. The key point here is that in doing so we can relate the long time behavior of a Cauchy problem with random coefficients with that of an associated (deterministic) Cauchy problem supported on a different "union" structure. We are not going to elaborate on this functorial viewpoint, but content ourselves with discussing in Sect. 5 a different, more sophisticated setting where the same principle can be seen in action. While in our two main applications we focus for the sake of simplicity on heat equations, our abstract theory is by no means restricted to this scope: general reaction-diffusion equations, evolution equations driven by poly-harmonic operators, or even systems switching between these two classes could, for example, be studied as well, see Remark 2.14. An easy application to a heat equation (on a fixed interval) with random boundary conditions is sketched in Example 2.1.

The case of combinatorial graphs is tightly related to the topic of random walk in random environments, see e.g. the classical surveys by Zeitouni [39,40], which roughly speaking describe the behavior of a random walker who at each step finds herself moving in a new realization of a $d$-dimensional bond-percolation graph. We also mention the connection to the somehow dual approach in [23], where Hussein and the third author develop a theory of evolution equations whose time domain is a (given!) tree-like metric graph: on each branch of the tree a different parabolic equation is considered. In comparison, in the present paper we restrict to the easiest possible case (the tree is simply $\mathbb{R}_{+}$), but its branches can have random length.

At the same time, if the evolution of $\mathcal{L}(t)$ is, in fact, deterministic, then (1.1) is essentially a non-autonomous evolution equation; well-posedness theory of such problems is a classical topic of operator theory, while some criteria for exponential stability have been recently obtained in [2] in the context of diffusion on metric graphs: in comparison with ours, the conditions therein are much more restrictive in that each realization of the considered graph is assumed to be connected.

The convergence of piecewise deterministic Markov processes (or random switching system) is discussed, in particular concerning the ergodicity of the Markov processes [5,11]. The results in [8] are concerned with the non-ergodicity of a switching system in the fast jump rate regime and open the path to similar results in [27].

\section{Setting of the Problem and Main Results}

In this section we introduce a general setting for abstract random evolution problems: we will successively show that our motivating problem $(\mathbf{P})$ is but one special instance of a system that can be described in this way.

To begin with, we construct the random mechanism of switching by means of a semi-Markov process. These processes have been introduced by Levy [28] and Smith [36] in order to overcome the limitation induced by the exponential distribution of the 
jump-time intervals and developed by Pyke $[33,34]$. These models are widely used in the literature to model random evolution problems and, more generally, evolution in random media, see e.g. Korolyuk [26].

Let $(Z(t), t \geq 0)$ be a semi-Markov process taking values in a set $E$, which denotes a given set of indices, defined on a suitable probability space $(\Omega, \mathcal{F}, \mathbb{P})$. By definition, this means that there exists a Markov renewal process $\left\{\left(X_{n}, \tau_{n}\right): n \in \mathbb{N}\right\}$, where $\left\{X_{n}\right\}$ is a Markov chain with values in $E$ and $\left\{\tau_{n}\right\}$ are the renewal times between jumps. The distribution function of $\tau_{n}$ depends on the state of the Markov process $X_{n}$ and, conditioned on $X_{n}=x$, it is given by

$$
\Phi_{x}(t)=\mathbb{P}\left(\tau_{n+1}<t \mid X_{n}=x\right)=q(x, E, t), \quad t \geq 0 .
$$

If we denote $\theta_{x}$ the renewal time in the state $x$ (i.e., the time spent in $x$ before the next jump) then the distribution function of $\theta_{x}$ is just $\Phi_{x}(t)$.

If we introduce the counting process $N(t):=\max \left\{n: T_{n} \leq t\right\}$, then $Z(t)=X_{N(t)}$. The joint distribution is given by the transition probability function $q(x, y, t)$

$$
q(x, y, t)=\mathbb{P}\left(X_{n+1}=y, \tau_{n+1}<t \mid X_{n}=x\right) .
$$

By definition, for fixed $t,(x, y) \mapsto q(x, y, t)$ is a sub-Markovian transition function, i.e.,

$$
q(x, y, t) \geq 0 \text { and } \sum_{z \in E} q(x, z, t) \leq 1 \quad \text { for all } x, y \in E \text { and all } t \geq 0 .
$$

The non-negative random variables $\tau_{n}$ define the time intervals between jumps, while the Markov renewal times $\left\{T_{n}, n \in \mathbb{N}\right\}$ defined by

$$
T_{0}=0, \quad T_{n}=\sum_{k=1}^{n} \tau_{k}, \quad n \in \mathbb{N}
$$

are the regeneration times.

For simplicity, in the sequel we assume that random variables $\left\{\tau_{n}\right\}$ are independent and the distribution of $\tau_{n+1}$ only depends on the state of the Markov chain $X_{n}=x$. Therefore, the transition probability function can be represented in the form

$$
q(x, y, t)=\pi(x, y) \Phi_{x}(t)
$$

where $(\pi(x, y))$ is a Markov transition matrix and $\Phi_{x}(t)$ are, for any $x \in E$, the probability distribution functions of the renewal time in the state $x$.

Clearly, Markov chains and Markov processes with discrete state space are examples of semi-Markov processes (the first is associated with $\tau_{n} \equiv 1$, the second with independent, exponentially distributed $\tau_{n}$ ). Our standing probabilistic assumptions are summarized in the following. 
Assumption 2.1 $Z=(Z(t))_{t \geq 0}$ is a semi-Markov process based on a Markov renewal process $\left\{\left(X_{n}, \tau_{n}\right): n \in \mathbb{N}\right\}$ over the state space $E \times[0, \infty)$ such that

(1) the Markov transition matrix $(\pi(x, y))$ defines an irreducible Markov chain with finite state space $E=\{1, \ldots, N\}$;

(2) the inter-arrival times $\tau_{n}$ are either constant, or their distribution functions $\Phi_{x}(t)$, for every $x \in E$, have a finite continuous density function $\phi_{x}(t)>0$ for a.e. $t>0$; and

(3) the inter-arrival times $\tau_{n}$ have finite expected value $\mathbb{E}^{x}\left[\tau_{n}\right]=\mu_{x}>0$.

The sequence of jump times associated to the process $Z$ is $T_{0}=0, T_{n+1}=T_{n}+\tau_{n+1}$ for $n \geq 0$.

Remark 2.2 Since the embedded Markov process $X$ is irreducible, there exists a unique invariant distribution $\rho=\left(\rho_{1}, \ldots, \rho_{N}\right)$ for it.

Moreover, this implies that the total time spent in any state by the semi-Markov process $Z$ is infinite almost surely, and the fraction of time spent in $x \in E=\{1, \ldots, N\}$ satisfies

$$
\Theta_{x}:=\lim _{t \rightarrow \infty} \frac{1}{t} \int_{0}^{t} \mathbb{1}_{\{Z(s)=x\}} \mathrm{d} s=\frac{\rho_{x} \mu_{x}}{\sum_{j \in E} \rho_{j} \mu_{j}} .
$$

Once our random environment has been described, we can introduce the evolution problem.

We consider an ensemble $\mathcal{K}=\left\{A_{1}, \ldots, A_{N}\right\}$ of linear operators on a normed space $H$; clearly, the cardinality of $\mathcal{K}$ is the same as that of the state space $E$ of the Markov chain.

We can now introduce the abstract random Cauchy problem

$$
\left\{\begin{aligned}
\frac{d}{d t} u(t) & =A(Z(t)) u(t), \\
u(0) & =f
\end{aligned}\right.
$$

where $A(Z(t))=A_{X_{n}}$ for $t \in\left[T_{n}, T_{n+1}\right)$. The solution of (2.2) is a random process, where the stochasticity enters the picture through the semi-Markov process $(Z(t))_{t \geq 0}$. Notice that (1.1) is a special case of (2.2) on the finite-dimensional space $H=\mathbb{C}^{\bar{d}}$.

In the literature, (deterministic) non-autonomous Cauchy problems of the form (2.2) are a classical topic with a well-developed theory, see e.g. [3,15,37]. In this paper, we shall use the following natural modification of the notion of solution.

Definition 2.3 Assume that there exists a finite partition $0=T_{0}<T_{1}<\ldots<T_{N}=$ : $T$ of $[0, T]$ such that $A(Z(t))=A_{X_{n}}$ for all $t \in\left[T_{n-1}, T_{n}\right), n=1, \ldots, N$. We say that a càglàd function $u:[0, T] \rightarrow H$ is a solution of (2.2) on $[0, T]$ if

(1) $u \in C^{1}\left(\left(T_{n-1}, T_{n}\right) ; H\right)$ for all $n=1, \ldots, N$;

(2) $u(t) \in D\left(A_{X_{n}}\right)$ for all $t \in\left(T_{n-1}, T_{n}\right)$ and $n=1, \ldots, N$;

(3) $u^{\prime}(t)=A_{X_{n}} u(t)$ for all $t \in\left(T_{n-1}, T_{n}\right)$ and $n=1, \ldots, N$.

Sufficient conditions for well-posedness of (2.2) are given by the following. 
Assumption 2.4 $H$ is a separable, complex Hilbert space and for every $j \in\{1, \ldots, N\}$ the closed, densely defined operator $A_{j}: D\left(A_{j}\right) \subset H \rightarrow H$ generates a stronglycontinuous, analytic semigroup of contractions and it has no spectral values on $i \mathbb{R}$, with the possible exception of 0 .

Definition 2.5 A solution $u$ for (2.2) is a stochastic process $\{u(t), t \geq 0\}$ which is required to be adapted to the filtration $\left\{\mathcal{F}_{t}\right\}$ generated by the SMP $Z$ :

$$
\mathcal{F}_{t}:=\sigma\left\{\left\{\tau_{n} \leq t\right\} \cap\left\{\left(x_{0}, \ldots, x_{n}\right) \in B\right\}, n \in \mathbb{N}, B \in E^{n+1}\right\}
$$

and whose trajectories solve the identity $u^{\prime}(t)=A(Z(t)) u(t)$ almost surely in the sense of Definition 2.3.

Existence and uniqueness of the solution in the sense of previous definition is a consequence of the well-posedness of the Cauchy problem driven by the operator $A_{X_{n}}$ on the time interval $\left(T_{n-1}, T_{n}\right)$.

Theorem 2.6 Under the Assumptions 2.1 and 2.4, given $f \in H$, (2.2) has a unique solution $u$, which can be expressed as $u(t)=S(t) f$ in terms of the random propagator $(S(t))_{t \geq 0} \subset \mathcal{L}(H)$ defined by

$$
S(t):=e^{\left(t-T_{n}\right) A_{X_{n}}} \prod_{k=0}^{n-1} e^{\left(T_{k+1}-T_{k}\right) A_{X_{k}}}, \quad t \in\left[T_{n}, T_{n+1}\right), n \in \mathbb{N} .
$$

In particular, $u$ is a continuous function (almost surely).

Proof Let us fix a path of the semi-Markov process $Z$, which is identified by the sequences of states $\left\{X_{n}\right\}$ and times $\left\{T_{n}\right\}$. By standard arguments (see e.g. [18]) there exists a unique solution $u(t)$ of the Cauchy problem in $\left[0, T_{1}\right)$ with leading operator $A_{X_{0}}$ : this solution has limit $u\left(T_{1}\right)=\lim _{t \uparrow T_{1}} u(t)$. Next, we consider the Cauchy problem in $\left[T_{1}, T_{2}\right)$ with leading operator $A_{X_{1}}$ and initial condition $u\left(T_{1}\right)$. Notice that the solution is continuous in $T_{1}$. Again, there exists a unique solution which has limit in $T_{2}$. We can repeat this argument: since by assumption $\lim T_{n}=+\infty$ almost surely, we obtain the thesis.

We notice the following equivalent expression of the random propagator $(S(t))_{t \geq 0}$ in terms of the inter-arrival times $\left\{\tau_{n}\right\}$ and the counting process $(N(t))_{t \geq 0}$ introduced above:

$$
S(t):=e^{\left(t-T_{N(t)}\right) A_{X_{N(t)}}} \prod_{k=0}^{N(t)-1} e^{\tau_{k+1} A_{X_{k}}}, \quad t \in \mathbb{R}_{+} .
$$

After establishing well-posedness of our abstract random Cauchy problem, we are interested in studying the long-time behavior of its solutions. To this purpose, we are going to impose the following.

Assumption $2.7 A_{j}$ has compact resolvent for every $j \in\{1, \ldots, N\}$. 
It follows from the Assumptions 2.4 and 2.7 that each $A_{j}$ has finite-dimensional null space, hence a fortiori

$$
K:=\bigcap_{j=1}^{N} \operatorname{ker} A_{j}
$$

is finite-dimensional, too. If $k:=\operatorname{dim} K>0$, then we denote by $\left\{e_{1}, \ldots, e_{k}\right\}$ an orthonormal basis of $K$.

We shall throughout denote by $P_{K}$ the orthogonal projector onto $K$ and $P_{j}$ the projector onto ker $A_{j}$. In general, for a projector $P$, its orthogonal operator is $P^{\perp}:=I-$ $P$. For the sake of consistency of notation, we use the same notation also in the case $K=\{0\}$.

Remark 2.8 In particular, it holds that $A_{j} e_{i}=0$, for all $j=1, \ldots, N$ and all $i=$ $1, \ldots, k$. Since the range of $P_{K}$ is spanned by null vectors of $A_{j}$ for each $j=1, \ldots, N$, $P_{K}$ commutes with each $A_{j}$, each semigroup operator $e^{t A_{j}}$, and each spectral projector $P_{j}$ onto $\operatorname{ker} A_{j}, j=1, \ldots, N, t \geq 0$.

Remark 2.9 Let $A$ be an operator which satisfies our Assumptions 2.4 and 2.7. Notice that they require $A$ to be dissipative and, thanks to Assumption 2.7, the spectrum of $A$ is discrete. By [18, Cor. IV.3.12 and Cor. V.2.15] there exists a spectral decomposition $H=H_{0} \oplus H_{d}$ where $H_{0}=\operatorname{ker}(A)$ and $H_{d}=H_{0}^{\perp}$ and the restriction of $A$ to $H_{d}$ generates an analytic contraction semigroup with strictly negative growth bound $s_{d}(A)=\sup \{\Re(\lambda): \lambda \in \sigma(A) \backslash\{0\}\}<0$.

In order to examine the long time behavior of the solution, we introduce a notion of convergence in the almost sure sense.

Definition 2.10 We say that a random propagator $(S(t))_{t \geq 0} \subset \mathcal{L}(H)$ converges in norm $\mathbb{P}$-almost surely towards a (deterministic) operator $M \in \mathcal{L}(H)$ if

$$
\mathbb{P}\left(\|\cdot\|-\lim _{t \rightarrow \infty} S(t)=M\right)=1
$$

Next result provides the main result about the asymptotic behaviour of the random propagator $(S(t))_{t \geq 0}$ associated to the Cauchy problem (2.2).

Theorem 2.11 Under the Assumptions 2.1, 2.4, and 2.7 the random propagator $(S(t))_{t \geq 0}$ for the Cauchy problem (2.2) converges in norm $\mathbb{P}$-almost surely towards the orthogonal projector $P_{K}$ onto $K:=\bigcap_{j=1}^{N} \operatorname{ker} A_{j}$.

Let us finally discuss the asymptotic behavior of the random evolution problem (2.2) under an additional assumption that is inspired by a result from [2], where nonautonomous diffusion equations on a fixed network are studied. Our aim is to study when the solution converges exponentially, for all initial data $f$, towards the orthogonal projector of $f$ onto the eigenspace with respect to the simple eigenvalue 0 . Adapting the ideas of [2] to our general setting, we shall impose the following. 
Assumption 2.12 There is one operator, say $A_{1}$, whose null space ker $A_{1}$ is contained in the null space of all other operators $A_{2}, \ldots, A_{N}$. In this case, we have that $K:=$ $\bigcap_{j=1}^{N} \operatorname{ker} A_{j}=\operatorname{ker} A_{1}$.

It turns out that under this additional assumption $(S(t))_{t \geq 0}$ converges in norm exponentially fast towards the orthogonal projector $P_{K}$ onto $K$. We stress that this is again a probabilistic assertion, since the result depends on the path of the systemactually, on the number of visits to the state corresponding to $A_{1}$.

Theorem 2.13 Under the assumptions of Theorem 2.11, let additionally the Assumption 2.12 hold. Then the almost sure convergence of the random propagator towards $P_{K}$ is exponential with rate $\alpha$, where

$$
\alpha \geq \lim _{t \rightarrow+\infty} \frac{1}{t} \int_{0}^{t}\left(-s_{d}\left(A_{1}\right)\right) \mathbb{1}_{\left(Z(s)=A_{1}\right)} \mathrm{d} s=\left(-s_{d}\left(A_{1}\right)\right) \Theta_{1}>0,
$$

and $s_{d}\left(A_{1}\right)$, introduced in Remark 2.9, is strictly negative thanks to the Assumption 2.12 and $\Theta_{1}$ was introduced in (2.1).

We postpone the proofs of our main results to Sect. 3 .

Remark 2.14 1) The Assumption 2.4 is especially satisfied if each $A_{j}$ is self-adjoint and negative semi-definite. In this case, moreover, $s_{d}\left(A_{j}\right)=\lambda_{k_{j}+1}\left(A_{j}\right)$ the largest non-zero eigenvalue, where $k_{j}$ is the dimension of $\operatorname{ker}\left(A_{j}\right)$ (we denote $\lambda_{k}$ the sequence of eigenvalues of $A_{j}$, and w.l.o.g. we assume that they are ordered: $\lambda_{1} \geq \lambda_{2} \geq \ldots$; then $\lambda_{k}=0$ for all $k<k_{j}$ ).

There are, however, further classes of operators satisfying it. If the semigroup generated by $A_{j}$ is positive and irreducible, for example, it follows from the Krel̆nRutman Theorem that the generator's spectral bound is a simple, isolated eigenvalue. A more general class of examples can be found invoking the theory of eventually positive semigroups, see [13, Thm. 8.3].

2) If we strengthen Assumption 2.12 by requiring that all operators in the ensamble $\mathcal{K}$ satisfy $\operatorname{ker}\left(A_{j}\right)=K$, then the statement of Theorem 2.13 becomes deterministic in the sense that the convergence towards the orthogonal projector $P_{K}$ always occurs.

\subsection{Randomly Switching Heat Equations}

The scope of our result is not restricted to graphs and networks. To illustrate this, we consider a toy model - a heat equation with initial data $f \in L^{2}(0,1)$, under different boundary conditions - where the switching takes place at the level of operators, rather than underlying structures. Here we show that convergence to the projector onto the intersection of the null spaces holds. A more complex example, where the thermostat model with switching in the boundary conditions, is given in [27]: in that case, nonergodicity is possible under certain conditions on the parameters.

(1) We first consider two different realizations $A_{1}, A_{2}$ of the Laplacian acting on $L^{2}(0,1)$ : with Neumann and with Krein-von Neumann boundary conditions, which lead to the domains 


$$
D\left(A_{1}\right):=\left\{u \in H^{2}(0,1): u^{\prime}(0)=u^{\prime}(1)=0\right\}
$$

and

$$
D\left(A_{2}\right):=\left\{u \in H^{2}(0,1): u^{\prime}(0)=u^{\prime}(1)=u(1)-u(0)\right\}
$$

respectively, [35, Exa. 14.14]. Both operators satisfy the Assumption 2.4. Furthermore, the null space of the former realization is one-dimensional, as it consists of the constant functions; whereas a direct computation shows that null space of the latter realization is 2-dimensional, as it consists of all affine functions on $[0,1]$; hence the intersection $K$ of both null spaces is spanned by the constant function $\mathbb{1}$ on $(0,1)$. Both associated heat equations are well-posed, yet the latter is somewhat exotic in that the governing semigroup is not sub-markovian. We are interested in the long-time behavior of this mixed system (2.2), with $A(Z(t)) \in\left\{A_{1}, A_{2}\right\}$ : if the switching obeys the rule in the Assumption 2.1, the random propagator $(S(t))_{t \geq 0}$ converges in norm $\mathbb{P}$-almost surely towards the orthogonal projector onto the intersection of both null spaces, i.e., onto the space of constant functions on $[0,1]$; hence the solution of the abstract random Cauchy problem (2.2) converges $\mathbb{P}$-almost surely towards the mean value of the initial data $f \in L^{2}(0,1)$.

(2) On the other hand, if we aim at studying the switching between Dirichlet and Neumann boundary conditions, and thus introduce the realization $A_{3}$ with domain

$$
D\left(A_{3}\right):=\left\{u \in H^{2}(0,1): u(0)=u(1)=0\right\},
$$

then one sees the intersection space $K$ is trivial, as ker $A_{3}=\{0\}$, hence the random propagator converges in norm $\mathbb{P}$-almost surely to 0 if the Assumption 2.1 is satisfied.

(3) Also observe that upon perturbing $A_{3}$ we find the new operator

$$
\begin{aligned}
\tilde{A}_{3} u & :=A_{3} u+\pi^{2} u \\
D\left(\tilde{A}_{3}\right) & :=D\left(A_{3}\right),
\end{aligned}
$$

whose null space is now one-dimensional, as it is spanned by $\sin (\pi \cdot)$. Nevertheless, ker $A_{1} \cap \operatorname{ker} \tilde{A}_{3}=\{0\}$, hence again under the Assumption 2.1 the system switching between $A_{1}, \tilde{A}_{3}$ converges towards 0 .

(4) Finally, let us consider a switching between $A_{1}$ and $A_{4}$ defined as

$$
\begin{aligned}
A_{4} u & :=\frac{d}{d x}\left(p \frac{d u}{d x}\right) \\
D\left(A_{4}\right) & :=D\left(A_{1}\right),
\end{aligned}
$$

where $p \in W^{1, \infty}(0,1), p(x)>0$ for all $x \in[0,1]$. Because ker $A_{1}$ and $\operatorname{ker} A_{4}$ both agree with the space of constant functions, under the Assumption 2.1 the random propagator converges in norm $\mathbb{P}$-almost surely towards the orthogonal projector onto the space of the constant functions. 
Moreover, as a consequence of Theorem 2.13 we can observe the exponential convergence of the random propagator for some (but not all) of these toy models. In particular, this holds whenever we take $A_{3}$ in the ensemble $\mathcal{K}$ : indeed, we have $K=\{0\}$ and then the Assumption 2.12 is satisfied, since the first eigenvalue $\lambda_{1}^{(3)}$ of $A_{3}$ is strictly negative. The exponential convergence of $(S(t))_{t \geq 0}$ can be shown also for randomly switching systems where $\mathcal{K} \subset\left\{A_{1}, A_{2}, A_{4}\right\}$. In all of these cases $K$ agrees with the space of constant functions on $(0,1)$, hence it is one-dimensional and the Assumption 2.12 is still fulfilled, since the second eigenvalue $\lambda_{2}^{(j)}<0$, for $j=1,4$. On the other hand, we cannot apply Theorem 2.13 and then prove the exponential convergence of the random propagator for all those models which switch $\tilde{A}_{3}$ with $A_{1}$ or/and $A_{2}$ or/and $A_{4}$. In fact, this implies that the intersection space $K$ is trivial again, but no one operator has strictly negative first eigenvalue.

\section{Technical Lemmas and Proofs}

\subsection{A Monotonicity Lemma}

The following lemma 3.4 provides the crucial tool to prove the assertion of Theorem 2.11. It shows how we can bound the norm of the random product of matrices which generates the random propagator $(S(t))_{t \geq 0}$ with respect to the stopping times.

Let $L \geq N$ and $\left(k_{1}, \ldots, k_{L}\right)$ be a sequence of indices that covers the whole $E=$ $\{1, \ldots, N\}$. Given an ensemble $\mathcal{K}$ of operators satisfying the Assumption 2.4, let us consider the associated sequence of operators $\left(A_{k_{1}}, \ldots, A_{k_{L}}\right)$ taken from $\mathcal{K}$. We shall denote $P_{j}$ the projection on the kernel ker $A_{j}$ and $P_{K}$ the projection on $K=$ $\cap_{j=1}^{L} \operatorname{ker} A_{k_{j}}=\cap_{i=1}^{N} \operatorname{ker} A_{i}$.

Remark 3.1 In the proof we will need some known results in functional analysis: if $T$ is a compact operator on a reflexive Banach space $X$, then there exists $x$ belonging to the unit sphere of $X$ such that $\|T\|=\|T x\|$, i.e., the norm of $T$ is attained: see e.g. [1, Cor. 1]. This is in particular true if $T=T(t)$ for some $t>0$, provided the semigroup generated by $A$ is analytic (or even merely norm continuous) and $A$ has compact resolvent, see [18, Thm. II.4.29]. Finally, we will need the well-known fact that the compact operators form a two-side ideal in in the space $\mathcal{L}(H)$ of bounded linear operators on $H$.

The following results are necessary steps in order to prove the main result of this section.

Lemma 3.2 Let $(T(t))_{t \geq 0}$ be a contractive, analytic strongly continuous semigroup on a Hilbert space $H$ whose generator A has compact resolvent and no eigenvalue on the imaginary axis, with the possible exception of 0 . Then the following assertions hold:

(1) $\left\|T\left(t^{\prime}\right) x\right\|<\|T(t) x\|$ for all $x \notin \operatorname{ker} A$ and all $t^{\prime}>t \geq 0$;

(2) $\operatorname{ker} A=\left\{x \in H:\left\|T\left(t_{0}\right) x\right\|=\|x\|\right\}$ for some $t_{0}>0$. 
Proof (1) Fix $x \notin \operatorname{ker} A$ and let $0 \leq t<t^{\prime}$.

Let us first consider the case of injective $A$, so that $P=0$, where we denote by $P$ the orthogonal projector onto $\operatorname{ker} A$. Then $x \neq 0$, and $T(t) x \rightarrow 0$ as $t \rightarrow \infty$ by the Jacobs-deLeeuw-Glicksberg theory, see [18, Thm. V.2.14 and Cor. V.2.15]. Due to analyticity of the semigroup, the mapping $\varphi:(0, \infty) \ni t \mapsto\|T(t) x\|^{2} \in \mathbb{R}$ is real analytic: indeed, for each $x \in H$ the mapping $(0, \infty) \ni t \mapsto T(t) x \in H$ is real analytic, hence it can be represented by an absolutely converging power series, say $T(t) x=\sum_{k=0}^{\infty} t^{k} f_{k}$; but then, the Cauchy product of $\sum_{k=0}^{\infty} t^{k} f_{k}$ with itself, given by $\sum_{m=0}^{\infty} t^{m} \sum_{l=0}^{m}\left(f_{l}, f_{m-l}\right)$, is absolutely converging towards $\|T(t) x\|^{2}=$ $(T(t) x, T(t) x)$.

If $\|T(t) x\|=\left\|T\left(t^{\prime}\right) x\right\|$, then $\varphi$ is constant on the interval $\left[t, t^{\prime}\right]$ : indeed, by contractivity of the semigroup

$$
\|T(s) x\| \leq\|T(t) x\|=\left\|T\left(t^{\prime}\right) x\right\| \leq\|T(s) x\| \quad \text { for all } s \in\left[t, t^{\prime}\right] .
$$

Due to the identity theorem for real analytic functions, $\varphi$ is now constant on $(0, \infty)-\mathrm{a}$ contradiction, since $\varphi(t) \rightarrow\|x\|^{2} \neq 0$ as $t \searrow 0$, but $\varphi(t) \rightarrow 0$ as $t \rightarrow \infty$. This proves the theorem in case that $P=0$.

Let us now consider the case of general $P$ : observe that $P x \neq x$, since $x \notin \operatorname{ker} A$. Applying the first step of the proof to the restriction of $(T(t))_{t \geq 0}$ to the $H \ominus \operatorname{ker} A$, we see that

$$
\|T(t)(I-P) x\|^{2}>\left\|T\left(t^{\prime}\right)(I-P) x\right\|^{2}
$$

hence by Pythagoras' theorem

$$
\begin{aligned}
\|T(t) x\|^{2} & =\|T(t) P x\|^{2}+\|T(t)(I-P) x\|^{2} \\
& >\|T(t) P x\|^{2}+\left\|T\left(t^{\prime}\right)(I-P) x\right\|^{2} \\
& =\left\|T\left(t^{\prime}\right) P x\right\|^{2}+\left\|T\left(t^{\prime}\right)(I-P) x\right\|^{2}=\left\|T\left(t^{\prime}\right) x\right\|^{2}
\end{aligned}
$$

where the second to last identity holds because the fixed space of $(T(t))_{t \geq 0}$

$$
\operatorname{fix}(T(t))_{t \geq 0}:=\{x \in H: T(t) x=x \text { for all } t \geq 0\}
$$

agrees with the null space of its generator $A$ by [18, Cor. IV.3.8], hence $T(t) y=y$ for all $y \in \operatorname{ker} A$ and all $t \geq 0$.

(2) We see that

$$
\begin{aligned}
\operatorname{fix}(T(t))_{t \geq 0} & \subset\{x \in H:\|T(t) x\|=\|x\| \text { for all } t \geq 0\} \\
& \subset\left\{x \in H:\left\|T\left(t_{0}\right) x\right\|=\|x\| \text { for some } t_{0} \geq 0\right\} \\
& \stackrel{(1)}{\subset} \operatorname{ker} A .
\end{aligned}
$$

This concludes the proof, since as recalled before ker $A=\operatorname{fix}(T(t))_{t \geq 0}$. 
The following is probably linear algebraic folklore, but we choose to give a proof since we could not find an appropriate reference.

Lemma 3.3 Let $H$ be a Hilbert space and let $P_{1}, \ldots, P_{m}$ be finitely many orthogonal projectors on $H$; let $P_{K}$ be the orthogonal projector onto $K:=\bigcap_{i=1}^{m} \operatorname{rg} P_{i}$. If $P_{i}$ is compact for at least one $i=1, \ldots, L$, then the operator $P_{m} \ldots P_{1} P_{K}^{\perp}$ has norm strictly less than 1:

$$
\left\|P_{m} \ldots P_{1} P_{K}^{\perp}\right\|=1-\varepsilon<1 .
$$

Proof It is obvious that $\left\|P_{m} \ldots P_{1} P_{K}^{\perp}\right\| \leq 1$. We proceed by contradiction and assume that

$$
\left\|P_{m} \ldots P_{1} P_{K}^{\perp}\right\|=1 .
$$

Since at least one $P_{i}$ is compact, so is the whole product, hence it is norm-attainable, see Remark 3.1: there exists $x \in H$ with $\|x\|=1$ such that $\left\|P_{m} \ldots P_{1} P_{K}^{\perp} x\right\|=\|x\|=1$. Notice that

$$
1=\left\|P_{m} \ldots P_{1} P_{K}^{\perp} x\right\| \leq\left\|P_{m} \ldots P_{1}\right\|\left\|P_{K}^{\perp} x\right\|
$$

hence $\left\|P_{K}^{\perp} x\right\|=1=\|x\|$ and it follows that $x=P_{K}^{\perp} x$. We then substitute in previous equality and get

$$
1=\left\|P_{m} \ldots P_{1} P_{K}^{\perp} x\right\|=\left\|P_{m} \ldots P_{1} x\right\|
$$

and the same reasoning implies $\left\|P_{1} x\right\|=1$, and $x=P_{1} x$. Reiterating the same argument we obtain $x=P_{j} x$ for any $j=1, \ldots, m$, therefore $x \in K$; but we have $x=P_{K}^{\perp} x$, which implies $x=0$, a contradiction to $\|x\|=1$. Therefore, (3.2) is false and the thesis follows.

We now proceed to prove the main result of this section. Recall that the operators $A_{j}$ are assumed to be dissipative and $P_{j}$ is the projection on $\operatorname{ker} A_{j}$.

Lemma 3.4 Under the Assumptions 2.4 and 2.7, let $L \geq N$ and $\left(k_{1}, \ldots, k_{L}\right)$ be a sequence of indices that covers the whole $E=\{1, \ldots, N\}$. For $\eta>0$ small enough there exists $\delta>0$ such that, for $t_{i} \geq \delta>0, i=1, \ldots, L$, we have

$$
\left\|P_{K}^{\perp} e^{t_{L} A_{k_{L}}} \cdots e^{t_{1} A_{k_{1}}}\right\| \leq 1-\eta<1 .
$$

Proof Recall that $P_{i}$ is the orthogonal projection on $\operatorname{ker} A_{i}, K=\cap_{i=1}^{N} \operatorname{ker}\left(A_{i}\right)$, and the projection on $K$ satisfies $P_{K} P_{i}=P_{K}=P_{i} P_{K}, P_{K}^{\perp} P_{i}^{\perp}=P_{i}^{\perp}=P_{i}^{\perp} P_{K}^{\perp}$. Recall that Young's inequality for product implies that $a b \leq \frac{a^{2}}{2 \varepsilon}+\frac{\varepsilon b^{2}}{2}$ for every $\varepsilon>0$; we further simplify by noticing that $2 \varepsilon>\varepsilon / 2$, hence the first term on the right is bounded by $\frac{a^{2}}{\varepsilon / 2}$. In the sequel, we shall repeatedly use this estimate in the form: for all $\alpha>0$, $(a+b)^{2} \leq(1+\alpha) a^{2}+\left(1+\alpha^{-1}\right) b^{2}$. 
We have

$$
\begin{aligned}
\left\|e^{t_{L} A_{k_{L}}} \ldots e^{t_{2} A_{k_{2}}} e^{t_{1} A_{k_{1}}} P_{K}^{\perp} x\right\|^{2}= & \left\|e^{t_{L} A_{k_{L}}} \ldots e^{t_{2} A_{k_{2}}} e^{t_{1} A_{k_{1}}}\left(P_{k_{1}}+P_{k_{1}}^{\perp}\right) P_{K}^{\perp} x\right\|^{2} \\
\leq & (1+\alpha)\left\|e^{t_{L} A_{k_{L}}} \ldots e^{t_{2} A_{k_{2}}} e^{t_{1} A_{k_{1}}} P_{k_{1}} P_{K}^{\perp} x\right\|^{2} \\
& +\left(1+\alpha^{-1}\right)\left\|e^{t_{L} A_{k_{L}}} \ldots e^{t_{2} A_{k_{2}}} e^{t_{1} A_{k_{1}}} P_{k_{1}}^{\perp} P_{K}^{\perp} x\right\|^{2} \\
\leq & (1+\alpha)\left\|e^{t_{L} A_{k_{L}}} \ldots e^{t_{2} A_{k_{2}}} P_{k_{1}} P_{K}^{\perp} x\right\|^{2} \\
& +\left(1+\alpha^{-1}\right)\left\|e^{t_{L} A_{k_{L}}} \ldots e^{t_{2} A_{k_{2}}}\right\|^{2}\left\|e^{t_{1} A_{k_{1}}} P_{k_{1}}^{\perp} x\right\|^{2}
\end{aligned}
$$

where we use the fact that $e^{t_{1} A_{k_{1}}} P_{k_{1}} x=P_{k_{1}} x$ for any $x \in H, t_{1} \geq 0$, and that ker $A_{k_{1}} \supset K$, so $\left(\operatorname{ker} A_{k_{1}}\right)^{\perp} \subset K^{\perp}$; the first estimate follows from Young's inequality. Notice further that all semigroups involved are contraction operators, hence $\left\|e^{t_{L} A_{k_{L}}} \ldots e^{t_{2} A_{k_{2}}}\right\|^{2} \leq 1$; finally, we have $\left\|e^{t_{1} A_{k_{1}}} P_{k_{1}}^{\perp} x\right\| \leq e^{-t_{1} s_{d}\left(A_{k_{1}}\right)}\left\|P_{k_{1}}^{\perp} x\right\| \leq$ $e^{-t_{1} s_{d}\left(A_{k_{1}}\right)}\|x\|$. We recall that $s_{d}\left(A_{j}\right)$ is the growth bound of the operator $A_{j}$ on $\left(\operatorname{ker} A_{j}\right)^{\perp}$, see Remark 2.9, and in our assumptions $s_{d}\left(A_{j}\right)<0$. It follows that

$$
\begin{aligned}
& \left\|e^{t_{L} A_{k_{L}}} \ldots e^{t_{2} A_{k_{2}}} e^{t_{1} A_{k_{1}}} P_{K}^{\perp} x\right\|^{2} \leq(1+\alpha)\left\|e^{t_{L} A_{k_{L}}} \ldots e^{t_{2} A_{k_{2}}} P_{K}^{\perp} P_{k_{1}} x\right\|^{2} \\
& \quad+\left(1+\alpha^{-1}\right) e^{-2 t_{1} s_{d}\left(A_{k_{1}}\right)}\|x\|^{2} .
\end{aligned}
$$

We continue by splitting the first term in the right hand side

$$
\begin{aligned}
&\left\|e^{t_{L} A_{k_{L}}} \ldots e^{t_{2} A_{k_{2}}} e^{t_{1} A_{k_{1}}} P_{K}^{\perp} x\right\|^{2} \\
& \leq(1+\alpha)\left\|e^{t_{L} A_{k_{L}}} \ldots e^{t_{2} A_{k_{2}}}\left(P_{k_{2}}+P_{k_{2}}^{\perp}\right) P_{K}^{\perp} P_{k_{1}} x\right\|^{2} \\
&+\left(1+\alpha^{-1}\right) e^{-2 t_{1} s_{d}\left(A_{k_{1}}\right)}\|x\|^{2} \\
& \leq(1+\alpha)^{2}\left\|e^{t_{L} A_{k_{L}}} \ldots e^{t_{2} A_{k_{2}}} P_{k_{2}} P_{K}^{\perp} P_{k_{1}} x\right\|^{2} \\
&+\left(1+\alpha^{-1}\right)\left\|e^{t_{L} A_{k_{L}}} \ldots e^{t_{2} A_{k_{2}}} P_{k_{2}}^{\perp} P_{K}^{\perp} P_{k_{1}} x\right\|^{2} \\
& \quad+\left(1+\alpha^{-1}\right) e^{-2 t_{1} s_{d}\left(A_{k_{1}}\right)}\|x\|^{2} \\
& \leq(1+\alpha)^{2}\left\|e^{t_{L} A_{k_{L}}} \ldots e^{t_{3} A_{k_{3}}} P_{k_{2}} P_{K}^{\perp} P_{k_{1}} x\right\|^{2} \\
&+\left(1+\alpha^{-1}\right)\left\|e^{t_{L} A_{k_{L}}} \ldots e^{t_{3} A_{k_{3}}}\right\|^{2} e^{-2 t_{2} s_{d}\left(A_{k_{2}}\right)}\left\|P_{k_{2}}^{\perp} P_{K}^{\perp} P_{k_{1}} x\right\|^{2} \\
&+\left(1+\alpha^{-1}\right) e^{-2 t_{1} s_{d}\left(A_{k_{1}}\right)}\|x\|^{2} \\
& \leq(1+\alpha)^{2}\left\|e^{t_{L} A_{k_{L}}} \ldots e^{t_{3} A_{k_{3}}} P_{K}^{\perp} P_{k_{2}} P_{k_{1}} x\right\|^{2} \\
&+\left(1+\alpha^{-1}\right)\left(e^{-2 t_{2} s_{d}\left(A_{k_{2}}\right)}+e^{-2 t_{1} s_{d}\left(A_{k_{1}}\right)}\right)\|x\|^{2}
\end{aligned}
$$

and by recursion, we finally obtain

$$
\begin{aligned}
& \left\|e^{t_{L} A_{k_{L}}} \ldots e^{t_{2} A_{k_{2}}} e^{t_{1} A_{k_{1}}} P_{K}^{\perp} x\right\|^{2} \leq(1+\alpha)^{L}\left\|P_{k_{L}} \ldots P_{k_{3}} P_{k_{2}} P_{k_{1}} P_{K}^{\perp} x\right\|^{2} \\
& \quad+\left(1+\alpha^{-1}\right) \sum_{i=1}^{L} e^{-2 t_{i} s_{d}\left(A_{k_{i}}\right)}\|x\|^{2}
\end{aligned}
$$


The operator in the first term is bounded in norm by $1-\varepsilon$, thanks to Lemma 3.3; therefore, we obtain the estimate

$$
\left\|e^{t_{L} A_{k_{L}}} \ldots e^{t_{2} A_{k_{2}}} e^{t_{1} A_{k_{1}}} P_{K}^{\perp} x\right\|^{2} \leq\left((1+\alpha)^{L}(1-\varepsilon)^{2}+\left(1+\alpha^{-1}\right) \sum_{i=1}^{L} e^{-2 t_{i} s_{d}\left(A_{k_{i}}\right)}\right)\|x\|^{2}
$$

The thesis follows by first taking $\alpha$ small enough such that the first addendum is bounded by $1-2 \eta$, then taking $\delta$ large enough such that the second addendum is bounded by $\eta{ }^{1}$

Notice that in previous lemma there is not any a priori bound on the required $\delta$, which can be arbitrarily large. However, in the case of a fixed, deterministic clock, the same result follows for arbitrary $\delta>0$ however small.

Lemma 3.5 Under the Assumptions 2.4 and 2.7 , let $L \geq N$ and $\left(k_{1}, \ldots, k_{L}\right)$ be a sequence of indices that covers the whole $E=\{1, \ldots, N\}$. Then for all $\delta>0$

$$
\left\|P_{K}^{\perp} e^{\delta A_{k_{L}}} \cdots e^{\delta A_{k_{1}}}\right\|<1
$$

Proof Let us now prove the inequality by contradiction: because all semigroups as well as the projector $P_{K}^{\perp}$ are contractive and hence certainly $\left\|P_{K}^{\perp} e^{\delta A_{k_{L}}} \cdots e^{\delta A_{k_{1}}}\right\| \leq 1$, it suffices to assume that

$$
\left\|P_{K}^{\perp} e^{\delta A_{k_{L}}} \cdots e^{\delta A_{k_{1}}}\right\|=1
$$

since the product operator is a compact operator, as stated before, there would then exist some $x \in H, x \neq 0$, with $\left\|P_{K}^{\perp} e^{\delta A_{k_{L}}} \cdots e^{\delta A_{k_{1}}} x\right\|=\|x\|$. Because

$$
\left\|P_{K}^{\perp} e^{\delta A_{k_{L}}} \cdots e^{\delta A_{k_{1}}} x\right\| \leq\left\|P_{K}^{\perp} e^{\delta A_{k_{L}}} \cdots e^{\delta A_{k_{2}}}\right\|\left\|e^{\delta A_{k_{1}}} x\right\| \leq\left\|e^{\delta A_{k_{1}}} x\right\|,
$$

it follows that $\left\|e^{\delta A_{k_{1}}} x\right\|=\|x\|$ and hence, by Lemma 3.2.(2), $x \in \operatorname{ker} A_{k_{1}}$, i.e., $e^{\delta A_{k_{1}} x}=x$. Proceeding recursively we see that $x \in \bigcap_{i=1}^{L} \operatorname{ker} A_{k_{i}} \subset K$, whence

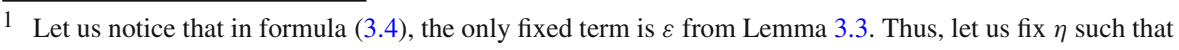

$$
\eta<\frac{1-(1-\varepsilon)^{2}}{2}=\frac{\varepsilon(2-\varepsilon)}{2}
$$

Then, we choose $\alpha$ such that

$$
(1+\alpha)^{L}(1-\varepsilon)^{2}=(1-2 \eta)
$$

and, setting $\lambda_{+}=\max \left\{s_{d}\left(A_{i}\right),: i=1, \ldots, L\right\}<0$, we may choose

$$
\delta>\frac{1}{|\lambda+|} \log \left(\eta^{-1} L\left(1+\alpha^{-1}\right)\right) .
$$


$e^{\delta A_{k_{i}}} x=x$ for all $i$ and hence

$$
\|x\|=\left\|P_{K}^{\perp} e^{\delta A_{k_{L}}} \cdots e^{\delta A_{k_{1}}} x\right\|=\left\|P_{K}^{\perp} x\right\|=0,
$$

a contradiction.

\subsection{Proof of Theorem 2.11}

We aim to apply Lemma 3.4 , hence we start by fixing a sequence $\xi=\left(\xi_{0}, \ldots, \xi_{L-1}\right)$ of states in $E$ which covers $E$ and is admissible for the sequence $X$, meaning that the probability that $X$ passes from the successive elements of $\xi$ in the given order is positive. Since $X$ is irreducible, we can also require that the transition $\xi_{L-1} \rightarrow \xi_{0}$ has positive probability.

Next step is to prove that, almost surely, the sequence $\xi$ occur infinitely times in the path of $X$ and that the waiting times are longer than $\delta$. Then the theorem follows as a consequence of our construction.

In the sequel, the constant $L$ is fixed and given by the length of the sequence $\xi$.

It is known that an irreducible Markov chain with finite state space $\left\{X_{n}\right\}$ is recurrent, i.e., every state is visited infinitely times, with a finite mean waiting time between successive visits. In the sequel, we need to prove that also any admissible cycle is recurrent.

An admissible cycle is a finite sequence of states of fixed length $L$ which returns to the starting point with positive probability. Formally, we require that $\xi=\left(\xi_{0}, \xi_{1}, \ldots, \xi_{L-1}\right)$ is an admissible cycle if

- the Markov chain follows this cycle with a strictly positive probability:

$$
p_{\xi_{0}, \xi_{1}} \cdots p_{\xi_{L-2}, \xi_{L-1}} p_{\xi_{L-1}, \xi_{0}}>0
$$

- by a suitable rotation of the indexes, it is always possible to let $\xi_{0}=X_{0}$.

Now, we can divide the path of $X$ in blocks of length $L: Y(0)=\left(X_{0}, \ldots, X_{L-1}\right)$, $Y(1)=\left(X_{L}, \ldots, X_{2 L-1}\right), \ldots$, and consider the stochastic process $Y$ taking values in a subset $\Lambda$ of $E^{L}$, where $\Lambda=\left\{\theta=\left(\theta_{0}, \ldots, \theta_{L-1}\right) \in E^{L}: \prod p_{\theta_{i}, \theta_{i+1}}>0\right\}$.

$$
\mathrm{Y} \text { is a Markov chain on the state } \operatorname{space} \Lambda \subset E^{L} \text {. }
$$

Proof In order to prove Markov property, we exploit the Markov property of the process $X$ and we obtain

$$
\begin{aligned}
\mathbb{P}(Y(n+1) & =\theta(n+1) \mid Y(0)=\theta(0), \ldots, Y(n)=\theta(n)) \\
& =\mathbb{P}\left(X_{(n+1) L}=\theta_{n+1 ; L}, \ldots, X_{(n+2) L-1}=\theta_{n+1 ; L-1} \mid Y_{0}\right. \\
& \left.=\theta_{0 ; 0}, X_{1}=\theta_{0 ; 1}, \ldots, X_{(n+1) L-1}=\theta_{n ; L-1}\right) \\
& =\mathbb{P}\left(X_{(n+1) L}=\theta_{n+1 ; L}, \ldots, X_{(n+2) L-1}=\theta_{n+1 ; L-1} \mid Y_{0}\right. \\
& \left.=\theta_{0 ; 0}, X_{1}=\theta_{0 ; 1}, \ldots, X_{(n+1) L-1}=\theta_{n ; L-1}\right)
\end{aligned}
$$




$$
\begin{aligned}
& =\mathbb{P}\left(X_{(n+1) L}=\theta_{n+1 ; L}, \ldots, X_{(n+2) L-1}=\theta_{n+1 ; L-1} \mid Y_{n L}\right. \\
& \left.=\theta_{n ; 0}, \ldots, X_{(n+1) L-1}=\theta_{n ; L-1}\right) \\
& =\mathbb{P}(Y(n+1)=\theta(n+1) \mid Y(n)=\theta(n)) .
\end{aligned}
$$

We shall denote $\bar{p}=\left(\bar{p}_{\theta, \eta}\right)_{\theta, \eta \in \Lambda}$ the transition matrix associated to the Markov chain $Y$.

Let $d$ be the period of the Markov chain $X(d=1$ if the chain is aperiodic). We notice first that $d \mid L$. If $d>1$, the state space $E$ can be divided into $p$ sub-classes $E_{0}, \ldots, E_{d-1}$, such that $\cup E_{i}=E$, and $E_{i} \cap E_{j}=\emptyset$ for $i \neq j$. For an aperiodic irreducible chain, we can set $E=E_{0}$. It holds that $p^{n}(x, y)>0$ only if $x \in E_{i}$ and $y \in E_{i+n}$ (where all the indices are taken modulus $d$ ) and $p^{n d}(x, y)>0$ for all sufficiently large $n$ and for all $x, y$ in the same class $E_{r}$.

The Markov chain $Y$ inherits an analog division, i.e., $\Lambda=C_{0} \cup \cdots \cup C_{d-1}$, where $C_{i}=\left\{\theta=\left(\theta_{0}, \ldots, \theta_{L-1}\right) \in \Lambda, \mid \theta_{0} \in E_{i}\right\}$. Assume that there exists $n$ such that $\bar{p}^{n}(\theta, \eta)>0$. Then $\theta_{0} \in E_{i}, \theta_{L-1} \in E_{i+L-1}=E_{i-1}$ (since $L$ is a multiple of $d$, hence $i+L-1 \cong i-1(\bmod d)), \eta_{0} \in E_{i+n L}=E_{i}$. If $d>1$, previous computation implies that $Y$ is no longer irreducible; however, if one consider the restriction of $Y$ to any of the classes $C_{i}$, we have the following result.

Proposition 3.6 If $X$ is a homogeneous, irreducible Markov chain of period $d$, then $Y$ restricted to any of the classes $C_{i}(i+0, \ldots, d-1)$ is a homogeneous, irreducible, aperiodic Markov chain on the given class (if $X$ is aperiodic, i.e. $d=1$, then $Y$ is irreducible and aperiodic on the whole $\Lambda$ ).

Proof Let $\theta$ and $\eta$ in the same class $C_{i}$, which implies that $\theta_{L-1} \in E_{i-1}$ and $\eta_{0} \in E_{i}$. Then there exists $n_{0}$ large enough such that for $n>n_{0}, p^{n d+1}\left(\theta_{L-1}, \eta_{0}\right)>0$. Taking $n>n_{0}$ in such a way that $k=n d / L$ is integer, we get that $\bar{p}^{k}(\theta, \eta)>0$, but since $\theta$ and $\eta$ are arbitrary, this implies that the class $C_{i}$ is closed and irreducible. Moreover, since $(k+1) L=(n+L / d) d$ it follows that also $\bar{p}^{k+1}(\theta, \eta)>0$, which implies that $Y$ is aperiodic on the class $C_{i}$, and this concludes the proof.

Corollary 3.7 Assume that X is a homogeneous, irreducible Markov chain. Then any admissible state y for the Markov chain $Y$ is recurrent.

Let $\xi=\left(\xi_{0}, \ldots, \xi_{L-1}\right)$ be the admissible cycle fixed at the beginning of the proof. Recall the representation of $S(t)$ given in (2.4), and take $t=n L-1$ for simplicity:

$$
\left\|P_{K}^{\perp} S(n L-1)\right\| \leq\left\|P_{K}^{\perp} \prod_{k=0}^{n L-1} e^{\tau_{k+1} A_{X_{k}}}\right\| \leq \prod_{m=0}^{n}\left\|P_{K}^{\perp} e^{\tau_{(m+1) L} A_{X_{(m+1) L-1}}} \ldots e^{\tau_{m L+1} A_{X_{m L}}}\right\|
$$

Let $N(n)=\sum_{k=0}^{n} \mathbb{1}_{Y_{k}=\xi} \mathbb{1}_{\tau_{k L+1} \geq \delta \ldots} \ldots \mathbb{1}_{\tau_{k(L+1)} \geq \delta}$ be the number of visit up to time $n$ to the state $\xi$ by the Markov chain $Y$ introduced above, such that all the waiting times in the successive states are at least as long as $\delta$. Since the state $\xi$ is recurrent for the chain $Y$ and the events $\left\{\tau_{k L+1} \geq \delta\right\}$ are independent and have strictly positive probability, it follows that $N(n) \rightarrow \infty$ as $n \rightarrow \infty$, almost surely. 
Now, we notice that in the right hand side of (3.6) we have $N(n)$ terms which can be bounded, thanks to Lemma 3.4 , by $1-\eta<1$ and the remaining terms have norm bounded by 1 , hence

$$
\left\|P_{K}^{\perp} S(n L-1)\right\| \leq(1-\eta)^{N(n)} \stackrel{t \rightarrow+\infty}{\longrightarrow} 0
$$

By Remark 2.8 we can write the random propagator as

$$
S(t)=P_{K} S(t)+P_{K}^{\perp} S(t)=P_{K}+P_{K}^{\perp} S(t)
$$

and the thesis

$$
\lim _{t \rightarrow+\infty}\left\|S(t)-P_{K}\right\|=0 \quad \mathbb{P}-\text { a.s. }
$$

follows by (3.7).

\subsection{Proof of Theorem 2.13}

As done in the proof of Theorem 2.11, we can write the random propagator as

$$
S(t)=P_{K} S(t)+P_{K}^{\perp} S(t)
$$

and show that $\left\|P_{K}^{\perp} S(t)\right\| \rightarrow 0$ as $t \rightarrow+\infty$ in order to obtain the thesis.

Denote $u(t):=S(t) f$ for all initial data $f$; we can estimate the norm of the vector $P_{K}^{\perp} u(t) \in H$ by

$$
\begin{aligned}
\left\|P_{K}^{\perp} u(t)\right\|^{2}-\left\|P_{K}^{\perp} f\right\|^{2} & =\int_{0}^{t} \frac{d}{d s}\left\|P_{K}^{\perp} u(s)\right\|^{2} \mathrm{~d} s \\
& =2 \Re\left(\int_{0}^{t}\left(\frac{d}{d s} P_{K}^{\perp} u(s), P_{K}^{\perp} u(s)\right) \mathrm{d} s\right) \\
& =2 \int_{0}^{t} \Re\left(A(Z(s)) P_{K}^{\perp} u(s), P_{K}^{\perp} u(s)\right) \mathrm{d} s,
\end{aligned}
$$

where the last equality holds due to Remark 2.8 and because $\frac{d}{d s}$ and $P_{K}^{\perp}$ commute. We split the above integral with respect to the various states of $Z(t)$ :

$$
\left\|P_{K}^{\perp} u(t)\right\|^{2}-\left\|P_{K}^{\perp} f\right\|^{2}=\sum_{j=1}^{N} 2 \int_{0}^{t} \mathbb{1}_{(Z(s)=j)} \Re\left(A_{X_{j}} P_{K}^{\perp} u(s), P_{K}^{\perp} u(s)\right) \mathrm{d} s,
$$

and since all the $A_{k}$ 's are dissipative, we have the trivial estimate

$$
\left\|P_{K}^{\perp} u(t)\right\|^{2}-\left\|P_{K}^{\perp} f\right\|^{2} \leq 2 \int_{0}^{t} \mathbb{1}_{(Z(s)=1)} \Re\left(A_{1} P_{K}^{\perp} u(s), P_{K}^{\perp} u(s)\right) \mathrm{d} s .
$$


Now, by Assumption 2.12, $K=\operatorname{ker}\left(A_{1}\right)$ and, by Remark 2.9, $A_{1}$ restricted to $K^{\perp}$ has growth bound $s_{d}\left(A_{1}\right)$ strictly negative, hence the above estimate becomes

$$
\begin{aligned}
\left\|P_{K}^{\perp} u(t)\right\|^{2}-\left\|P_{K}^{\perp} f\right\|^{2} & \leq-2 s_{d}\left(A_{1}\right) \int_{0}^{t} \mathbb{1}_{(Z(s)=1)}\left(P_{K}^{\perp} u(s), P_{K}^{\perp} u(s)\right) \mathrm{d} s \\
& =-2 s_{d}\left(A_{1}\right) \int_{0}^{t} \mathbb{1}_{(Z(s)=1)}\left\|P_{K}^{\perp} u(s)\right\|^{2} \mathrm{~d} s .
\end{aligned}
$$

By Gronwall's Lemma we deduce that

$$
\left\|P_{K}^{\perp} u(t)\right\|^{2}=\left\|P_{k}^{\perp} S(t) f\right\|^{2} \leq\left\|P_{K}^{\perp} f\right\|^{2} e^{-2 s_{d}\left(A_{1}\right) \int_{0}^{t} \mathbb{1}_{(Z(s)=1)} \mathrm{d} s} .
$$

The thesis now follows from Remark 2.2: indeed, the integral diverges to $+\infty \mathbb{P}$ almost surely, hence $\left\|P_{K}^{\perp} S(t)\right\| \rightarrow 0$. Moreover, as stated in Remark 2.2, the fraction of time spent in a state by the semi-Markov process $Z$ can be computed to be

$$
\lim _{t \rightarrow+\infty} \frac{1}{t} \int_{0}^{t} \mathbb{1}_{\left(Z(s)=A_{1}\right)} \mathrm{d} s=\Theta_{1}>0,
$$

where $\Theta_{1}$ was introduced in Remark 2.2. It follows from (3.9) that the speed of convergence to 0 is at least equal to $\left(-2 s_{d}\left(A_{1}\right)\right) \Theta_{1}$.

\section{Combinatorial Graphs}

A simple (finite, undirected) combinatorial graph $G=(V, E)$ is a couple defined by a finite set $V$ of vertices $V$ and a subset $E \subset V^{(2)}$ of unordered pairs $e:=\{v, w\}$ of elements of $\mathrm{V}$; such a pair e is interpreted as the edge connecting the vertices $\mathrm{V}, \mathrm{w}$.

Given a simple graph $G=(V, E)$, let us introduce a positive weight function on the set of vertices $\mathrm{V}$

$$
m: \mathrm{V} \rightarrow(0,+\infty)
$$

which induces the scalar product

$$
(f, g)_{m}:=\sum_{\mathrm{v} \in \mathrm{V}} m(\mathrm{v}) f(\mathrm{v}) \overline{g(\mathrm{v})}, \quad f, g \in \mathbb{C}^{\mathrm{V}},
$$

on the space $\mathbb{C}^{\mathrm{V}}$ of complex valued functions $f: \mathrm{V} \rightarrow \mathbb{C}$ : we denote by $\ell_{m}^{2}(\mathrm{~V})$ the Hilbert space $\mathbb{C}^{\mathrm{V}}$ with respect to $(\cdot, \cdot)_{m}$. In addition, let

$$
\mu: \mathrm{E} \rightarrow(0,+\infty)
$$

be a positive weight function on the set of edges $\mathrm{E}$. We call the 4-tuple (V, E, $m, \mu)$ a weighted combinatorial graph. 
Remark 4.1 We stress that each weighted graph is a metric space with respect to the shortest path metric; while the topology does depend on the weights, any two weights define equivalent topologies, and in particular it does not depend on $m, \mu$ whether $\mathrm{G}$ is connected or not.

Let us recall the notion of discrete Laplacian (or Laplace-Beltrami matrix) $\mathcal{L}_{m, \mu}$ on a weighted graph $\mathrm{G}=(\mathrm{V}, \mathrm{E}, m, \mu)$, cf. [31, § 2.1.4]—or shortly: weighted Laplacian. For any vertex $\mathrm{v} \in \mathrm{V}$, let $\mathrm{E}_{\mathrm{V}}$ denote the set of all edges having $\mathrm{v}$ as an endpoint. Then $\mathcal{L}_{m, \mu}: \ell_{m}^{2}(\mathrm{~V}) \rightarrow \ell_{m}^{2}(\mathrm{~V})$ is defined by

$$
\mathcal{L}_{m, \mu} f(\mathrm{v}):=\frac{1}{m(\mathrm{v})} \sum_{\mathrm{e}=\{\mathrm{v}, \mathrm{w}\} \in \mathrm{E}_{\mathrm{v}}} \mu(\mathrm{e})(f(\mathrm{w})-f(\mathrm{v})), \quad \forall \mathrm{v} \in \mathrm{V} ;
$$

$\mathcal{L}_{m, \mu}$ reduces to the discrete, negative semi-definite Laplacian if $\mu \equiv 1$ and $m \equiv 1$; i.e., $\mathcal{L}_{1,1}$ is minus the Laplacian matrix that is common in the literature [31, $\left.\S 2.1 .4\right]$. Indeed, we stress that we have not adopted the usual sign convention of algebraic graph theory, as any such $\mathcal{L}_{m, \mu}$ is self-adjoint and negative semi-definite. More generally, $\mathcal{L}_{m, \mu}$ satisfies the Assumptions 2.4 and 2.7 and it can be shown that $\mathcal{L}_{m, \mu}$ (and not $-\mathcal{L}_{m, \mu}$ ) generates a Markovian semigroup. The associated sesquilinear form $q: \ell_{m}^{2}(\mathrm{~V}) \times \ell_{m}^{2}(\mathrm{~V}) \rightarrow \mathbb{C}$ is given by

$$
q(f, g)=\sum_{\mathrm{e}=\{\mathrm{v}, \mathrm{w}\} \in \mathrm{E}} \mu(\mathrm{e})(f(\mathrm{v})-f(\mathrm{w})) \overline{(g(\mathrm{v})-g(\mathrm{~W}))}, \quad f, g \in \ell_{m}^{2}(\mathrm{~V}),
$$

and satisfies

$$
q(f, g)=\left(\mathcal{L}_{m, \mu} f, g\right)_{m}=\left(f, \mathcal{L}_{m, \mu} g\right)_{m}, \quad f, g \in \ell_{m}^{2}(\mathrm{~V})
$$

accordingly, its Rayleigh quotient is

$$
\frac{\left(f, \mathcal{L}_{m, \mu} f\right)_{m}}{(f, f)_{m}}=\frac{q(f, f)}{\|f\|_{m}^{2}}=\frac{\sum_{\mathrm{e}=\{\mathrm{v}, \mathrm{w}\} \in \mathrm{E}} \mu(\mathrm{e})|f(\mathrm{v})-f(\mathrm{w})|^{2}}{\|f\|_{m}^{2}}, \quad f \neq 0 .
$$

It follows from (4.1) that $\lambda=0$ is an eigenvalue of each weighted Laplacian $\mathcal{L}_{m, \mu}$ : the associated eigenfunctions are constant on each connected component of $\mathrm{G}=$ $(\mathrm{V}, \mathrm{E}, m, \mu)$. Therefore, it turns out that the null space of $\mathcal{L}_{m, \mu}$ agrees with the null space of the unweighted Laplacian (on $(V, E)$ ) associated with $G$.

\subsection{The General Model}

Throughout this section we consider a finite collection $\mathcal{C}$ of graphs.

Assumption $4.2 \mathcal{C}=\left\{\mathrm{G}_{1}, \ldots, \mathrm{G}_{N}\right\}$, where $\mathrm{G}_{1}=\left(\mathrm{V}, \mathrm{E}_{1}, m_{1}, \mu_{1}\right), \ldots, \mathrm{G}_{N}=\left(\mathrm{V}, \mathrm{E}_{N}, m_{N}, \mu_{N}\right)$ are simple graphs with same vertex set $\mathrm{V}$ but possibly different edge sets $\mathrm{E}_{i}$, vertex weights $\mu_{i}$, and edge weights $\mu_{i}, i=1, \ldots, N$. 
The following seems to be natural but not quite standard: we prefer to note it explicitly.

Definition 4.3 (Union and intersection of weighted graphs) The union of $\mathrm{G}_{i}=$ $\left(\mathrm{V}, \mathrm{E}_{i}, m_{i}, \mu_{i}\right), i=1, \ldots, N$, is the weighted graph $\mathrm{G}_{\cup}=(\mathrm{V}, \mathrm{E}, m, \mu)$ with set of vertices $\mathrm{V}$, set of edges $\mathrm{E}:=\bigcup_{i=1}^{N} \mathrm{E}_{i}$, vertex weights

$$
m(\mathrm{v}):=\min _{i=1, \ldots, N} m_{i}(\mathrm{v}), \quad \mathrm{v} \in \mathrm{V},
$$

and edge weights

$$
\mu(\mathrm{e}):=\max _{i=1, \ldots, N} \mu_{i}(\mathrm{e}), \quad \mathrm{e} \in \mathrm{E} .
$$

Likewise, the intersection of $\mathrm{G}_{i}=\left(\mathrm{V}, \mathrm{E}_{i}, m_{i}, \mu_{i}\right), i=1, \ldots, N$, is the weighted graph $\mathrm{G}_{\cap}=(\mathrm{V}, \mathrm{E}, m, \mu)$ with set of vertices $\mathrm{V}$, set of edges $\mathrm{E}:=\bigcap_{i=1}^{N} \mathrm{E}_{i}$, vertex weights

$$
m(\mathrm{v}):=\max _{i=1, \ldots, N} m_{i}(\mathrm{v}), \quad \mathrm{v} \in \mathrm{V}
$$

and edge weights

$$
\mu(\mathrm{e}):=\min _{i=1, \ldots, N} \mu_{i}(\mathrm{e}), \quad \mathrm{e} \in \mathrm{E}
$$

here we set $\mu_{i}(\mathrm{e}):=0$ if e $\notin \mathrm{E}_{i}$.

In this way, it is possible to study the behavior of the intersection of the null spaces of the Laplacian operators $\mathcal{L}_{m_{k}, \mu_{k}}\left(\mathrm{G}_{k}\right)$ associated with the graphs in $\mathcal{C}$. This result seems interesting on its own, since it explicitly connects the geometry of the graph with the algebraic property of the Laplacian operator.

Lemma 4.4 Given $G_{1}, \ldots, G_{N}$ combinatorial graphs satisfying the Assumption 4.2, let $G$ be their weighted union graph (see Definition 4.3) and let $\mathcal{L}_{m, \mu}(G)$ be the discrete Laplacian on $G$. Then

$$
\operatorname{ker} \mathcal{L}(G)=\bigcap_{i=1}^{N} \operatorname{ker} \mathcal{L}_{m_{i}, \mu_{i}}\left(G_{i}\right)
$$

Proof It suffices to work with unweighted graphs; for simplicity, moreover, we only prove the case $N=2$. In general, suppose that $\mathrm{G}_{,} \mathrm{G}_{1}$ and $\mathrm{G}_{2}$ are written as disjoint unions of connected components:

$$
\mathrm{G}=\bigsqcup_{h=1}^{l} \mathrm{G}^{(h)}, \quad \mathrm{G}_{1}=\bigsqcup_{j=1}^{m} \mathrm{G}_{1}^{(j)}, \quad \mathrm{G}_{2}=\bigsqcup_{k=1}^{n} \mathrm{G}_{2}^{(k)}
$$


Any eigenfunction associated with the null eigenvalue of $\mathcal{L}$ shall be constant on any connected component of $\mathrm{G}$, hence $\mathcal{B}_{\mathrm{G}}=\left\{\mathbb{1}_{h}, h=1, \ldots, l\right\}$, where

$$
\forall \mathrm{v} \in \mathrm{V}: \quad \mathbb{1}_{h}(\mathrm{v}):= \begin{cases}1 & \text { if } \mathrm{v} \in \mathrm{G}^{(h)} \\ 0 & \text { otherwise },\end{cases}
$$

is a basis for $\operatorname{ker} \mathcal{L}(\mathrm{G})$. Similarly, $\mathcal{B}_{1}=\left\{\mathbb{1}_{1, j}, j=1, \ldots, m\right\}$ and $\mathcal{B}_{2}=\left\{\mathbb{1}_{2, k}, k=\right.$ $1, \ldots, n\}$ are a basis of $\operatorname{ker} \mathcal{L}\left(\mathrm{G}_{1}\right)$ and $\operatorname{ker} \mathcal{L}\left(\mathrm{G}_{2}\right)$, respectively. Hence, we only need to prove that for all $h=1, \ldots, l, \mathbb{1}_{h}$ is in the intersection of the null spaces in $\mathcal{C}$ and then extend the result to $\operatorname{ker} \mathcal{L}(\mathrm{G})$ by linearity. In particular, denoting

$$
\begin{aligned}
& C_{1}^{(h)}:=\left\{j \in\{1, \ldots, m\} \quad \mathrm{G}_{1}^{(j)} \subseteq \mathrm{G}^{(h)}\right\}, \\
& C_{2}^{(h)}:=\left\{k \in\{1, \ldots, n\} \quad \mathrm{G}_{2}^{(k)} \subseteq \mathrm{G}^{(h)}\right\},
\end{aligned}
$$

then by construction the function $\mathbb{1}_{h}$ will be

$$
\forall \mathrm{v} \in \mathrm{V}: \quad \mathbb{1}_{h}(\mathrm{v}):= \begin{cases}1 & \text { if } \mathrm{v} \in \mathrm{G}_{1}^{(j)}, j \in C_{1}^{(h)} \\ 0 & \text { otherwise, }\end{cases}
$$

and

$$
\forall \mathrm{v} \in \mathrm{V}: \quad \mathbb{1}_{h}(\mathrm{v}):= \begin{cases}1 & \text { if } \mathrm{v} \in \mathrm{G}_{2}^{(k)}, k \in C_{2}^{(h)}, \\ 0 & \text { otherwise, }\end{cases}
$$

thus $\mathbb{1}_{h} \in \operatorname{ker} \mathcal{L}\left(\mathrm{G}_{1}\right) \cap \operatorname{ker} \mathcal{L}\left(\mathrm{G}_{2}\right)$, in fact it can be written as linear combination of both bases $\mathcal{B}_{1}$ and $\mathcal{B}_{2}$ as

$$
\mathbb{1}_{h}=\sum_{j \in C_{1}^{(h)}} \mathbb{1}_{1, j} \quad \text { or } \quad \mathbb{1}_{h}=\sum_{k \in C_{2}^{(h)}} \mathbb{1}_{2, k} .
$$

On the other hand, given $f \in \operatorname{ker} \mathcal{L}\left(\mathrm{G}_{1}\right) \cap \operatorname{ker} \mathcal{L}\left(\mathrm{G}_{2}\right)$, we have

$$
f=\alpha_{1} \mathbb{1}_{1,1}+\cdots+\alpha_{m} \mathbb{1}_{1, m}
$$

and

$$
f=\beta_{1} \mathbb{1}_{2,1}+\cdots+\beta_{n} \mathbb{1}_{2, n},
$$

where $\mathcal{B}_{1}$ and $\mathcal{B}_{2}$ as above. Then, comparing the expressions (4.2) and (4.3), we get that

$$
\begin{aligned}
& \alpha_{j}=\beta_{k}=c_{1}, \quad \forall j \in C_{1}^{(1)}, \forall k \in C_{2}^{(1)}, \\
& \vdots \\
& \alpha_{j}=\beta_{k}=c_{l}, \quad \forall j \in C_{1}^{(l)}, \forall k \in C_{2}^{(l)},
\end{aligned}
$$


and $f$ can also be expressed in terms of $\mathcal{B}_{\mathrm{G}}$ as

$$
f=\sum_{h=1}^{l} c_{h} \mathbb{1}_{h}
$$

thus $f \in \operatorname{ker} \mathcal{L}(\mathrm{G})$.

As a corollary, we notice the following result, concerning the relation between the connectedness of the union graph $\mathrm{G}$ (see Remark 4.1) and the dimension of the kernel of the (weighted or unweighted) Laplacian operator.

Corollary 4.5 Given $G_{1}, \ldots, G_{N}$ combinatorial graphs satisfying the Assumption 4.2, let $G$ be their weighted union graph. Then

$$
\text { G is connected } \Longleftrightarrow \bigcap_{i=1}^{N} \operatorname{ker} \mathcal{L}_{m_{i}, \mu_{i}}\left(G_{i}\right)=\langle\mathbb{1}\rangle \text {. }
$$

Partially motivated by Corollary 4.5 , with a slight abuse of notation we adopt in the following the notation $\mathcal{L}_{i}:=\mathcal{L}_{m_{i}, \mu_{i}}\left(\mathrm{G}_{i}\right), i=1, \ldots, N$. Fixed a probability space $(\Omega, \mathcal{F}, \mathbb{P})$, let $(Z(t))_{t \geq 0}$ be a semi-Markov process on the state space $E=\{1, \ldots, N\}$ which satisfies Assumption 2.1. In this section, we shall consider the random Cauchy problem

$$
\left\{\begin{aligned}
\frac{d u}{d t}(t, \mathrm{v}) & =\mathcal{L}_{X_{k}} u(t, \mathrm{v}), & & \mathrm{v} \in \mathrm{V}, t \in\left[T_{k}, T_{k+1}\right), k \in \mathbb{N}, \\
u(0, x) & =f(\mathrm{v}), & & \mathrm{v} \in \mathrm{V},
\end{aligned}\right.
$$

where $\mathcal{L}_{X_{k}}$ is the discrete Laplace operator associated with the currently selected graph $\mathrm{G}_{X_{k}}$. The above equation is also known in the literature as the (random) discrete heat equation.

We can now state our main result in this section. We recall that the relevant operators $\mathcal{L}_{m, \mu}$ satisfy the Assumptions 2.4 and 2.7, hence the following is a direct consequence of Theorem 2.11.

Theorem 4.6 Let $(Z(t))_{t \geq 0}$ be a semi-Markov process and $\mathrm{C}$ be a family of graphs that satisfy the Assumptions 2.1 and 4.2, respectively. Then the random propagator $(S(t))_{t \geq 0}$ for the Cauchy problem (4.5) converges in norm $\mathbb{P}$-almost surely towards the orthogonal projector $P_{K}$ onto the space $K=\bigcap_{i=1}^{N} \operatorname{ker} \mathcal{L}_{i}$.

The limiting operator can be identified with the orthogonal projector onto the constant functions, provided $\mathrm{G}$ is connected.

Corollary 4.7 Under the assumptions of Theorem 4.6, $(S(t))_{t \geq 0}$ converges in norm $\mathbb{P}$-almost surely to $P_{0}$ if and only if the union graph $G$ is connected. 
Proof Corollary 4.5 implies that $P_{0}=P_{K}$ if and only if the union graph $\mathrm{G}$ is connected, while Theorem 4.6 implies the convergence of $S(t)$ towards $P_{K}$, hence the sufficiency and necessity of the condition.

In the last part of this section we present two special cases of evolution on combinatorial graphs where we discuss the relation between our result and the existing literature.

\subsection{Connected Graphs}

In this section we assume that all the graphs in $\mathcal{C}$ are connected. As we have already seen, this assumption is unnecessarily strong if we aim at solving $(\mathbf{P})$.

However, we are going to show an interesting link between our problem and the analysis of the so-called left-convergent product sets [14]. For simplicity, in this section we assume that $\tau_{n}=1$ for every $n$, hence $T_{n}=n$ and $Z(t)=Z(\lfloor t\rfloor)=X_{\lfloor t\rfloor}$.

A set $\mathcal{K}=\left\{M_{1}, \ldots, M_{N}\right\}$ of matrices is said to have the left-convergent product property, or simply to be an $L C P$ set, if for every sequence $\mathrm{j}=\left(j_{n}\right)_{n \in \mathbb{N}}$ taking values in $\{1, \ldots, N\}$ the infinite left-product $M_{\mathrm{j}}:=\prod_{k=0}^{\infty} M_{j_{k}}$ converges. Given two sequences $\mathrm{j}$ and $\mathrm{j}^{\prime}$, define the metric

$$
\mathrm{d}\left(\mathrm{j}, \mathrm{j}^{\prime}\right):=N^{-r} \quad \text { where } \mathrm{r} \text { is the first index such that } j_{r} \neq j_{r}^{\prime}
$$

and call the topology induced by $\mathrm{d}$ on $\mathbb{S}=\left\{\mathrm{j}=\left(j_{n}\right)_{n \in \mathbb{N}}, j_{n} \in\{1, \ldots, N\}\right\}$ as sequence topology on $\mathbb{S}$. It is known [17] that $\mathcal{K}$ is an LCP set if $\mathcal{K}$ is paracontracting, meaning that for some matrix norm

$$
M x \neq x \Rightarrow\|M x\|<\|x\| \quad \text { for all } M \in \mathcal{K} \text { and } x \in \mathbb{R}^{d}
$$

The issue of convergence of infinite products of matrices has been finally settled in a fundamental paper by Daubechies and Lagarias: in particular, see [14, Thm. 4.1 and Thm. 4.2] and also the erratum in [16].

Proposition 4.8 [14, Thm. 4.2] Let $\mathcal{K}$ be a finite set of $d \times d$ matrices. Then the following are equivalent.

(a) $\mathcal{K}$ is an LCP set whose limit function $\mathrm{j} \mapsto M_{\mathrm{j}}$ is continuous with respect to the sequence topology on $\mathbb{S}$.

(b) All matrices $M_{i}$ in $\mathcal{K}$ have the same eigenspace $E_{1}$ with respect to the eigenvalue 1 , this eigenspace is simple for all $M_{i}$, and there exists a vector space $V$ such that $\mathbb{C}^{d}=E_{1} \oplus V$ and such that if $P_{V}$ is the oblique projector onto $V$ away from $\mathrm{e}_{1}$, then $P_{V} \mathcal{K} P_{V}$ is an LCP set whose limit function is identically 0.

In particular, if $E_{1}$ is a 1-dimensional subspace, then the limit function $M$ is the projector onto this space. 
Now we can state this result in the setting of combinatorial graphs. Under the assumption of connectedness of all graphs, Theorem 2.11 states that $S(t)$ will converge to $P_{0}$ (the projector on the subspace $\langle\mathbb{1}\rangle$ of constant functions) no matter which sequence of graphs we follow in (4.5), thus it provides the same result as in the deterministic case treated in Proposition 4.8. We shall give in Lemma 4.9 an alternative proof to this result, which specializes to the notation of graph theory.

Lemma 4.9 Let $\mathcal{C}=\left\{G_{1}, \ldots, G_{N}\right\}$ be a finite family of connected graphs and $Z=$ $\left(X_{n}, \tau_{n}=1\right)$ be an irreducible Markov chain. Then for any path of the process $Z$ the limit

$$
\lim _{t \rightarrow+\infty}\left\|S(t)-P_{0}\right\|=0
$$

holds for the random evolution problem (4.5).

Proof In our assumptions, 0 is a simple eigenvalue of each Laplacian matrix $\mathcal{L}_{k}:=$ $\mathcal{L}\left(\mathrm{G}_{k}\right)$ with associated eigenvector $\mathbb{1}$.

The orthogonal operator $P_{0}^{\perp}$ is again an orthogonal projector operator with range $\langle\mathbb{1}\rangle^{\perp}$. Notice that $P_{0} S(t)=P_{0}$ because $\operatorname{rg} P_{0}=\langle\mathbb{1}\rangle$ is contained in

$$
\text { fix }\left(e^{t \mathcal{L}_{k}}\right)_{t \geq 0}:=\left\{x: \bigvee \rightarrow \mathbb{C}: e^{t \mathcal{L}_{k}} x=x \text { for all } t \geq 0\right\}
$$

for every $1 \leq k \leq N$. Therefore,

$$
S(t)=P_{0} S(t)+\left(I-P_{0}\right) S(t)=P_{0}+P_{0}^{\perp} S(t) \quad \text { for all } t \geq 0,
$$

and we can prove the assertion by showing that

$$
\lim _{t \rightarrow+\infty}\left\|P_{0}^{\perp} S(t)\right\|=0
$$

First of all, by definition $P_{0}^{\perp}$ is idempotent and commutes with the exponential matrix of every Laplace operator. Hence

$$
P_{0}^{\perp} S(t)=P_{0}^{\perp} e^{(t-k) \mathcal{L}_{X_{k}}} e^{\mathcal{L}_{X_{k-1}}} \cdots e^{\mathcal{L}_{X_{0}}}=P_{0}^{\perp} e^{(t-k) \mathcal{L}_{X_{k}}} P_{0}^{\perp} e^{\mathcal{L}_{X_{k-1}}} \cdots P_{0}^{\perp} e^{\mathcal{L}_{X_{0}}} .
$$

We claim that

$$
\text { each matrix } P_{0}^{\perp} e^{\mathcal{L}_{i}}, i=1, \ldots, N \text { has norm strictly less than } 1 \text {. }
$$

By the finiteness of $\mathrm{E}$, we denote by

$$
\delta:=\max \left\{\left\|P_{0}^{\perp} e^{\mathcal{L}_{i}}\right\| \quad i=1, \ldots, N\right\}<1 .
$$


For all $t>0$, let $k \in \mathbb{N}$ be such that $k \leq t<k+1$. By sub-multiplicativity of the matrix norm we have

$$
\begin{aligned}
& \left\|P_{0}^{\perp} S(t)\right\|=\left\|P_{0}^{\perp} e^{(t-k) \mathcal{L}_{X_{k}}} P_{0}^{\perp} e^{\mathcal{L}_{X_{k-1}}} \cdots P_{0}^{\perp} e^{\mathcal{L}_{X_{0}}}\right\| \\
& \leq\left\|P_{0}^{\perp} e^{(t-k) \mathcal{L}_{X_{k}}}\right\| \quad\left\|P_{0}^{\perp} e^{\mathcal{L}_{X_{k-1}}}\right\| \cdots\left\|P_{0}^{\perp} e^{\mathcal{L}_{X_{0}}}\right\| \\
& \leq\left\|P_{0}^{\perp} e^{\mathcal{L}_{X_{k-1}}}\right\| \cdots\left\|P_{0}^{\perp} e^{\mathcal{L}_{X_{0}}}\right\| \leq \delta^{k-1} .
\end{aligned}
$$

If $t \rightarrow+\infty$, then $k \rightarrow+\infty$ and we finally get

$$
\lim _{t \rightarrow+\infty}\left\|P_{0}^{\perp} S(t)\right\|=0
$$

which implies the thesis.

In order to complete the proof it remains to show that claim (4.8) holds. We have proved a more refined version of this claim in Lemma 3.4; however, in the current setting, the proof is straightforward. Let $\mathcal{L}$ denote the Laplacian operator for a connected graph G. By a direct computation we have for all $t>0$

$$
\begin{aligned}
\left\|\left(I-P_{0}\right) e^{t \mathcal{L}} f\right\|^{2} & =\sum_{k=2}^{d} e^{2 t \lambda_{k}}\left(f, e_{k}\right)_{\ell^{2}}^{2} \leq e^{2 t \lambda_{2}} \sum_{k=2}^{d}\left(f, e_{k}\right)_{\ell^{2}}^{2} \\
& =e^{2 t \lambda_{2}}\left\|\left(I-P_{0}\right) f\right\|^{2} \leq e^{2 t \lambda_{2}}\|f\|^{2},
\end{aligned}
$$

whence $\left\|\left(I-P_{0}\right) e^{\delta \mathcal{L}}\right\|^{2}<e^{2 \delta \lambda_{2}}<1$ since $\lambda_{2}<0$.

\subsection{Randomly Switching Combinatorial Graphs with Non-zero Second Eigenvalue}

The goal here is to apply our exponential convergence criteria to combinatorial graphs. Consider the random evolution problem (4.5); we are going to show the exponential convergence of the random propagator $(S(t))_{t \geq 0}$ provided that the following assumption holds:

Assumption 4.10 There exists one combinatorial graph in $\mathrm{C}$, say $\mathrm{G}_{1}$, such that each connected component of $\mathrm{G}_{j}, j \neq 1$, is contained in one of the connected components of $G_{1}$.

As shown in the proof of Lemma 4.4, a consequence of the assumption above is that $\operatorname{ker} \mathcal{L}_{1} \subseteq \operatorname{ker} \mathcal{L}_{j}, j \neq 1$, therefore the Assumption 2.12 is satisfied and we can directly apply Theorem 2.13 .

Corollary 4.11 Let $(Z(t))_{t \geq 0}$ be a semi-Markov process and $\mathrm{C}$ be a family of graphs that satisfy the Assumptions 2.1 and 4.2, respectively. Let additionally the Assumption 4.10 hold.

Then the random propagator $(S(t))_{t \geq 0}$ converges in norm $\mathbb{P}$-almost surely exponentially fast towards the orthogonal projector $P_{K}$ with an exponential rate no lower 
than

$$
\alpha=-\sum_{j=1}^{N} s_{d}\left(\mathcal{L}_{j}\right) \Theta_{j}
$$

that is the average of the values $s_{d}\left(\mathcal{L}_{j}\right)$ (introduced in Remark 2.9) with respect to the fraction of time $\Theta_{j}$ spent by the process $Z(t)$ in the various states. ${ }^{2}$

Proof The assertion follows from Theorem 2.13. Notice that the exponential rate can be computed by

$$
-\frac{1}{t} \int_{0}^{t} s_{d}\left(\mathcal{L}_{Z(s)}\right) \mathrm{d} s=-\sum_{j=1}^{N} \frac{1}{t} s_{d}\left(\mathcal{L}_{j}\right) \int_{0}^{t} \mathbb{1}_{\left(Z(s)=\mathrm{G}_{j}\right)} \mathrm{d} s
$$

which converges, as $t \rightarrow \infty$, to (compare Remark 2.2)

$$
\alpha=-\sum_{j=1}^{N} \Theta_{j} s_{d}\left(\mathcal{L}_{j}\right)=-\sum_{j=1}^{N} s_{d}\left(\mathcal{L}_{j}\right) \frac{\rho_{j} \mu_{j}}{\sum_{l=1}^{N} \rho_{l} \mu_{l}} .
$$

This concludes the proof.

Remark 4.12 Assume that $G_{1}$ is connected (then the Assumption 4.10 is verified). It follows that the union graph $\mathrm{G}$ is connected, too, hence the intersection space $K$ is one-dimensional and $P_{K}=P_{0}$ is the projection onto the space of constant functions on V. Moreover $s_{d}\left(\mathcal{L}_{1}\right)=\lambda_{2}\left(\mathcal{L}_{1}\right)<0$. Adapting the proof of [19, Cor. 3.2] (where the convention is adopted that $\mathcal{L}$ is positive semi-definite) we see that each of the discrete Laplacians $\mathcal{L}_{k}$ on the weighted combinatorial graph $\mathrm{G}_{k}$ has second largest eigenvalue $\lambda_{2}\left(\mathcal{L}_{k}\right):=\lambda_{2}\left(G_{k}\right) \in\left[\lambda_{2}\left(G_{\cup}\right), \lambda_{2}\left(G_{\cap}\right)\right]$, where $G_{\cup}, G_{\cap}$ are the union and intersection graph introduced in Lemma 4.4, respectively: therefore we conclude that the convergence to equilibrium for the randomly switching problem is not faster (resp., not slower) than in the case of the heat equation on $\mathrm{G}_{\cup}$ (resp., on $\mathrm{G}_{\cap}$; observe that $\mathrm{G}_{\cap}$ may however be disconnected, and hence $\lambda_{2}\left(\mathrm{G}_{\cap}\right)$ may vanish, even if all $\mathrm{G}_{k}$ are connected).

Estimates on the rate of convergence to equilibrium of the random propagator are readily available: it is well-known that, for a generic unweighted connected graph $\mathrm{G},-|\mathrm{V}| \leq \lambda_{2}(\mathrm{G}) \leq-2\left(1-\cos \frac{\pi}{|\mathrm{V}|}\right)$, where the second inequality is an equality if and only if $G$ is a path graph, see [19, 3.10 and 4.3]. It follows that $\lambda_{2}\left(\mathcal{L}_{k}\right) \in$ $\left[-|V|,-2\left(1-\cos \frac{\pi}{|V|}\right)\right]$ if in particular $G_{\cap}$ is connected; this gives an estimate on the convergence rate in Corollary 4.11.

\footnotetext{
${ }^{2}$ It is possible to explicitly compute $\Theta_{1}$ in terms of the invariant distribution $\rho=\left(\rho_{1}, \ldots, \rho_{N}\right)$ associated to the embedded Markov chain $X$ and the expected values of the jump times for the different states $\mu_{j}=\mathbb{E}^{j}\left[\tau_{1}\right]$ by the formula
}

$$
\Theta_{j}=\frac{\rho_{j} \mu_{j}}{\sum_{l=1}^{N} \rho_{l} \mu_{l}} .
$$




\section{Metric Graphs}

In this section we discuss the application of Theorem 2.11 to finite metric graphs. Roughly speaking, metric graphs are usual graphs (as known from discrete mathematics) whose edges are identified with real intervals - in this case, finitely many interval of finite length; loops and multiple edges between vertices are allowed. While this casual explanation is usually sufficient [6,31], for our purposes we will need a more formal definition. We are going to follow the approach and formalism in [30].

Let $\mathrm{E}$ be a finite set. Given some $\left(\ell_{\mathrm{e}}\right)_{\mathrm{e} \in \mathrm{E}} \subset(0, \infty)$, we consider the disjoint union of intervals

$$
\mathcal{E}:=\bigsqcup_{\mathrm{e} \in \mathrm{E}}\left[0, \ell_{\mathrm{e}}\right]:
$$

we adopt the usual notation $(x, \mathrm{e})$ for the element of $\mathcal{E}$ with $x \in\left[0, \ell_{\mathrm{e}}\right]$ and $\mathrm{e} \in \mathrm{E}$.

We can define on $\mathcal{E}$ a (generalized) metric by setting

$$
d_{\mathcal{E}}((x, \mathrm{e}),(y, \mathrm{f})):= \begin{cases}d_{\mathrm{e}}(x, y)=|x-y|, & \text { if } \mathrm{e}=\mathrm{f} \text { and } x, y \in\left[0, \ell_{\mathrm{e}}\right] \\ \infty, & \text { otherwise }\end{cases}
$$

Consider the set

$$
\mathcal{V}:=\bigsqcup_{\mathrm{e} \in \mathrm{E}}\left\{0, \ell_{\mathrm{e}}\right\}=\bigsqcup_{\mathrm{e} \in \mathrm{E}}\left\{(0, \mathrm{e}),\left(\ell_{\mathrm{e}}, \mathrm{e}\right)\right\}
$$

of endpoints of $\mathcal{E}$. Given any equivalence relation $\equiv$ on $\mathcal{V}$, we extend it to an equivalence relation on $\mathcal{E}$ as follows: two distinct elements $\left(x_{1}, \mathrm{e}_{1}\right) \neq\left(x_{2}, \mathrm{e}_{2}\right) \in \mathcal{E}$ belong to the same equivalence class in $\mathcal{E}$ if and only if they belong to the set of vertices $\mathcal{V}$ and they are equivalent with respect to the relation $\equiv$ on $\mathcal{V},\left(x_{1}, \mathrm{e}_{1}\right) \equiv\left(x_{2}, \mathrm{e}_{2}\right)$ : we denote this equivalence relation on $\mathcal{E}$ again by $\equiv$ and we call $\mathcal{G}:=\mathcal{E} / \equiv$ a metric graph, with $\mathrm{E}$ its set of edges and $\mathrm{V}:=\mathcal{V} / \equiv$ its set of vertices. So, a vertex $\mathrm{V} \in \mathrm{V}$ is by definition an equivalence class consisting of boundary elements from $\mathcal{E}$, like $(0, \mathrm{e})$ or $\left(\ell_{\mathrm{f}}, \mathrm{f}\right)$. Beyond our formalism, the equivalence relation on $V$ can be understood as follows: two elements of $\mathcal{V}$ belong to the same equivalence class and can hence be identified if they are endpoints of two adjacent edges corresponding to the same vertex of the underlying combinatorial graph: see [30, Rem. 1.7] for more details, which will however not be necessary in the present context.

Two edges e, $f \in E$ are said to be adjacent if one endpoint of $e$ and one endpoint of $f$ lie in the same equivalence class $v \in \mathrm{V}$ (i.e., if e, f share an endpoint, up to identification by $\equiv)$; in this case we write $\mathrm{e} \sim \mathrm{f}$. Also, two vertices $\mathrm{v}, \mathrm{w} \in \mathrm{V}$ are said to be adjacent if there exists some (not necessarily unique) e $\in E$ such that $\{x, y\}=\left\{(0, e),\left(\ell_{e}, e\right)\right\}$ for representatives $x$ of $v$ and $y$ of $w$ (i.e., if there is an edge whose endpoints are $v, w$, up to identification by $\equiv$ ); in this case we write $\mathrm{v} \sim \mathrm{w}$.

Let us stress that by definition a metric graph is uniquely determined by a family $\left(\ell_{\mathrm{e}}\right)_{\mathrm{e} \in \mathrm{E}}$ and an equivalence relation on $\mathcal{V}$; however, its metric structure is independent on the orientation of the edges! 
As a quotient of metric spaces, any metric graph is a metric space in its own right with respect to the canonical quotient metric defined by

$$
d_{\mathcal{G}}(\xi, \theta):=\inf \sum_{i=1}^{k} d_{\mathcal{E}}\left(\xi_{i}, \theta_{i}\right), \quad \xi, \theta \in \mathcal{G},
$$

where the infimum is taken over all $k \in \mathbb{N}$ and all pairs of $k$-tuples $\left(\xi_{1}, \ldots, \xi_{k}\right)$ and $\left(\theta_{1}, \ldots, \theta_{k}\right)$ with $\xi=\xi_{1}, \theta=\theta_{k}$, and $\theta_{i} \sim \xi_{i+1}$ for all $i=1, \ldots, k-1,[4$, Def. 3.1.12], where $\sim$ denotes the adjacency relation introduced before. We call $d_{\mathcal{G}}$ the path metric of $\mathcal{G}$. A metric graph is said to be connected if the path metric doesn't attain the value $\infty$; in other words, if any two points of $\mathcal{G}$ can be linked by a path. Along with this metric structure there is a natural measure induced by the Lebesgue measure on each interval; accordingly, we can introduce the spaces

$$
C(\mathcal{G}) \text { and } L^{2}(\mathcal{G})
$$

as well as

$$
H^{1}(\mathcal{G}):=\left\{f \in L^{2}(\mathcal{G}) \cap C(\mathcal{G}): f^{\prime} \in L^{2}(\mathcal{G})\right\} .
$$

Again, these definitions do not depend on the orientation of the metric graph; but the notation

$$
f(\xi):=f_{\mathrm{e}}(x):=f((x, \mathrm{e})), \quad \xi:=(x, \mathrm{e}),
$$

does.

On the graph $\mathcal{G}$ we aim to introduce a differential operator acting as the second derivative on the functions $f_{j}(x)$ on every edge $\mathrm{e}_{j}$; and possibly more general operators of the form

$$
A_{\max }:=f \mapsto \frac{d}{d x}\left(p \frac{d f}{d x}\right)
$$

for some elliptic coefficient $p \geq p_{0}>0$ of class $W^{1, \infty}, p_{0} \in \mathbb{R}$. While it is natural to require that $f_{\mathrm{e}} \in H^{2}\left(0, \ell_{\mathrm{e}}\right)$ for every edge e, taking $\bigoplus_{\mathrm{e} \in \mathrm{E}} H^{2}\left(0, \ell_{\mathrm{e}}\right)$ as domain only defines an operator acting on functions on $L^{2}(\varepsilon)$ : this is not sufficient in order to define a self-adjoint operator and suitable boundary conditions shall thus be imposed in order for $A_{\max }$ to satisfy the Assumption 2.4.

Each realization of the elliptic operator $A$ we are interested in is equipped with natural vertex conditions: for each element $u$ in its domain

- $u \in C(\mathcal{G})$, and in particular $u$ is continuous across vertices;

- $u$ satisfies the Kirchhoff condition at each vertex, namely

$$
\forall \mathrm{v} \in \mathrm{V}: \quad \sum_{\substack{\mathrm{e} \in \mathrm{E} \\(0, \mathrm{e}) \in \mathrm{v}}} p_{\mathrm{e}}(\mathrm{v}) u_{\mathrm{e}}^{\prime}(\mathrm{v})=\sum_{\substack{\mathrm{f} \in \mathrm{E} \\\left(\ell_{\mathrm{f}}, \mathrm{f}\right) \in \mathrm{v}}} p_{\mathrm{f}}(\mathrm{v}) u_{\mathrm{f}}^{\prime}(\mathrm{v}),
$$


i.e., the weighted sum of the inflows equals the weighted sum of the outflows.

(Observe that in any vertex with degree 1 the latter becomes a Neumann boundary condition; and that the case $p \equiv 1$ defines the usual Laplacian $\Delta$ with natural vertex conditions on the metric graph $\mathcal{G}$.)

We can now define the operator $A$ with natural vertex conditions on $\mathcal{G}$, i.e.

$$
\begin{aligned}
(A u)_{\mathrm{e}}(x) & :=\left(p_{\mathrm{e}}(x) u_{\mathrm{e}}^{\prime}(x)\right)^{\prime}, \\
D(A) & :=\left\{u \in C(\mathcal{G}) \cap \bigoplus_{\mathrm{e} \in \mathrm{E}} H^{2}\left(0, \ell_{\mathrm{e}}\right): u \text { satisfies }(\mathrm{Kc})\right\} .
\end{aligned}
$$

Let us summarize the main results we need in our construction for the operator $A$ with natural vertex conditions. They are part of a general, well-established theory, see e.g. [25, Thm. 2.5 and Cor. 3.3] for edgewise constant coefficients $p_{\mathrm{e}}$ and [31, Thm. 6.67] for the general case.

Proposition 5.1 The operator A with natural vertex conditions on $H=L^{2}(\mathcal{G})$ is densely defined, closed, self-adjoint, and negative semi-definite; it has compact resolvent.

Thus, the Assumptions 2.4 and 2.7 are satisfied and $A$ generates a contractive strongly continuous semigroup, denoted by $\left(e^{t A}\right)_{t \geq 0}$. Hence the abstract Cauchy problem

$$
\left\{\begin{array}{l}
\frac{d}{d t} u(t)=A u(t), \quad t>0, \\
u(0)=f
\end{array}\right.
$$

is well-posed: for every $f \in L^{2}(\mathcal{G})$ there exists a unique mild solution given by

$$
u(t):=e^{t A} f, \quad \forall t \geq 0
$$

Moreover, continuous dependence on the initial data holds. Because $A$ is self-adjoint and hence the semigroup is analytic, the solution $u$ is for all $f \in L^{2}(\mathcal{G})$ of class $C^{1}\left((0, \infty) ; L^{2}(\mathcal{G})\right) \cap C((0, \infty) ; D(A))$.

By Proposition 5.1, the spectrum of $A$ consists of negative eigenvalues of finite multiplicity and the spectral radius satisfies $s(A)=0 \in \sigma(A)$. The study of the complete spectrum is still an open problem: actually, only in few cases it is fully determined and in general just some upper and lower bounds on the eigenvalues are known. In this work, we are going to emphasize the following property of $\sigma(A)$, see [25, Theorem 4.3].

Proposition 5.2 Let $\mathcal{G}$ be a finite metric graph and denote by $\mathcal{G}^{(1)}, \ldots, \mathcal{G}^{(l)}$ its connected components. Then, the multiplicity of 0 as eigenvalue of the operator A with natural vertex conditions is $l$. In particular, the piecewise constant functions $\left\{\mathbb{1}_{h}\right\}_{h=1}^{l}$, where

$$
\mathbb{1}_{h}(x)= \begin{cases}1 & \text { if } x \in \mathcal{G}^{(h)} \\ 0 & \text { otherwise }\end{cases}
$$

for all $h=1, \ldots, l$, form a basis of $\operatorname{ker} A$. 


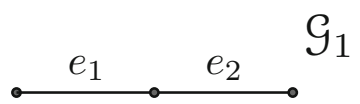

Fig. 1 Model A

\subsection{A Motivating Example}

Let us study on the interval $[0,2]$ the heat equation

$$
\left\{\begin{aligned}
\frac{\partial u}{\partial t}(t, x) & =\frac{\partial^{2} u}{\partial x^{2}} u(t, x), & & t \geq 0, x \in[0,2], \\
u(0, x) & =u_{0}(x), & & x \in[0,2],
\end{aligned}\right.
$$

where $u_{0} \in L^{2}(0,2)$. In particular, we are going to analyze two different and wellknown boundary value problems: in one case, we impose two Neumann conditions at $x=0$ and $x=2$, whereas the second setting keeps the same constraints at the boundaries, plus one additional Neumann condition at the middle point $x=1$.

Model A describes the evolution of the heat equation on [0,2] with Neumann boundary conditions in 0 and 2 . Formally, however, we consider [0,2] as the graph $\mathcal{G}_{1}$ with $\mathrm{V}=\{0,1,2\}$ and edges $\mathrm{e}_{1}=[0,1]$ and $e_{2}=[1,2]$.

The evolution is thus described by the Laplace operator $\Delta_{1}$ given by

$$
\begin{aligned}
D\left(\Delta_{1}\right) & =\left\{\begin{array}{l}
u=\left(u_{1}, u_{2}\right): \\
u_{1}^{\prime}(0)=u_{i} \in H^{2}(0,1), i=1,2,
\end{array}\right. \\
\Delta_{1} u & =\frac{d^{2} u}{d x^{2}} .
\end{aligned}
$$

The spectrum of $\Delta_{1}$ clearly agrees with that of the Laplacian with Neumann conditions on $[0,2]$, i.e.,

$$
\sigma\left(\Delta_{1}\right)=\left\{\lambda_{k}=-\frac{k^{2} \pi^{2}}{4}, k=0,1,2, \ldots\right\},
$$

with associated eigenfunctions

$$
\begin{aligned}
& e_{0}(x)=\frac{1}{\sqrt{2}}, x \in[0,2] \\
& e_{k}(x)=\cos \left(\frac{k \pi}{2} x\right), x \in[0,2], \quad k \geq 1
\end{aligned}
$$

In this way, for every initial condition $u_{0} \in L^{2}(0,2)$, we can explicitly write the solution in terms of the spectral representation

$$
u(t)=e^{t \Delta_{1}} u_{0}=\sum_{k=0}^{+\infty} e^{t \lambda_{k}}\left(u_{0}, e_{k}\right)_{L^{2}(0,2)} e_{k}
$$




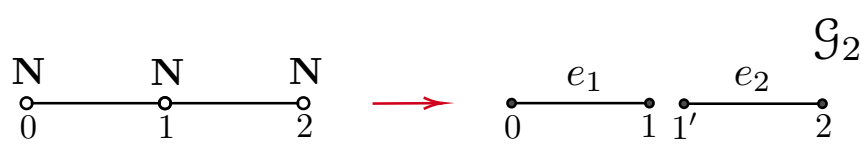

Fig. 2 Model B: on the right, the correct interpretation as a network equation with a disconnected graph

and, as expected, the limit distribution for long times agrees with the average of $u_{0}$ computed on the interval $[0,2]$

$$
\lim _{t \rightarrow+\infty} u(t)=\left(u_{0}, e_{0}\right) e_{0}=P_{0} u_{0}=\frac{1}{2} \int_{0}^{2} u_{0}(x) \mathrm{d} x .
$$

Model B describes the evolution of the heat equation on [0, 2] with Neumann boundary conditions in 0 , in 2 , as well as in 1 . Formally, we consider [0,2] as the graph $\mathcal{G}_{2}$ with $\mathrm{V}=\left\{0,1,1^{\prime}, 2\right\}$ and edges $e_{1}=[0,1]$ and $e_{2}=\left[1^{\prime}, 2\right]$.

The Laplace operator associated with $\mathcal{G}_{2}$ is $\Delta_{2}$ with domain

$$
\begin{aligned}
D\left(\Delta_{2}\right) & =\left\{u=\left(u_{1}, u_{2}\right) \in H^{2}(0,1) \oplus H^{2}\left(1^{\prime}, 2\right)\right. \\
& \left.: u_{1}^{\prime}(0)=u_{1}^{\prime}(1)=0, u_{2}^{\prime}\left(1^{\prime}\right)=u_{2}^{\prime}(2)=0\right\}, \\
\Delta_{2} u & =\frac{d^{2} u}{d x^{2}} .
\end{aligned}
$$

Here the dynamics is somehow different from the previous one: in fact, the Neumann condition placed in $x=1$ acts like an insulating "wall" through which heat exchanges are not allowed. The spectrum in this case is

$$
\sigma\left(\Delta_{2}\right)=\left\{\bar{\mu}_{k}=-k^{2} \pi^{2}, k=0,1,2, \ldots\right\}
$$

where every eigenvalue has now multiplicity two.

For every initial condition $g=\left(g_{1}, g_{2}\right) \in L^{2}(\mathcal{G})$ the solution $u(t)$ converges, as $t \rightarrow \infty$, to the vector-valued function whose two coordinates are the mean value of $g_{1}$ and $g_{2}$, respectively.

Starting from these two models, we now introduce the following scenario: imagine that we are going to study the heat diffusion along the interval $[0,2]$ with Neumann boundary conditions. However, at each renewal time $T_{n}$ we can decide to add or remove one third Neumann condition at $x=1$. In particular, the choice of considering three or two constraints is determined by a suitable random process. This means that the system switches between Model A and Model B and the stochastic evolution problem is of the form (2.2).

We shall see that the asymptotic behavior of our systems is given by the uniform $\mathbb{P}$ almost sure convergence towards the orthogonal projector $P_{0}$ to the constant functions. 


\subsection{The General Model}

Like in Sect. 4, we are going to introduce ensembles of metric graphs.

Assumption $5.3 \mathcal{C}=\left\{\mathcal{G}_{1}, \ldots, \mathcal{G}_{N}\right\}$, where $\mathcal{G}_{1}, \ldots, \mathcal{G}_{N}$ are metric graphs with the same edge set $\mathcal{E}$ (i.e., defined upon the same finite set $\mathrm{E}$ and the same vector $\left(\ell_{\mathrm{e}}\right)_{\mathrm{e} \in \mathrm{E}}$ ) but possibly different sets of vertices $\mathcal{V}_{1}:=\mathcal{V}\left(\mathcal{G}_{1}\right), \ldots, \mathcal{V}_{N}:=\mathcal{V}\left(\mathcal{G}_{N}\right)$ (i.e., the equivalence relations $\equiv_{1}, \ldots, \equiv_{N}$ may be different).

Once again, we introduce a probability space $(\Omega, \mathcal{F}, \mathbb{P})$ and a semi-Markov process $(Z(t))_{t \geq 0}$ satisfying Assumption 2.1.

At this point, we can associate with each graph $\mathcal{G}_{i}$ in $\mathcal{C}$ an operator $A_{i}$ with natural vertex conditions and elliptic coefficient $p_{i} \in W^{1, \infty}$ as in (5.2), which we denote by

$$
\left(A_{i}, D\left(A_{i}\right)\right), \quad i=1, \ldots, N:
$$

we emphasize that the different vertex sets induce different operator domains, even though all operators satisfy the same class of vertex conditions: for example, "cutting through a vertex", hence producing two vertices of lower degree out of a vertex of larger degree, induces a new operator with relaxed continuity conditions (and two new Kirchhoff conditions).

By Proposition 5.1, all these operators satisfy the Assumptions 2.4 and 2.7. We can state our main problem, i.e., the continuous random evolution on metric graphs

$$
\left\{\begin{array}{l}
\frac{d}{d t} u(t)=A_{X_{k}} u(t), \quad t \in\left[T_{k}, T_{k+1}\right), \\
u(0)=f \in L^{2}(\mathcal{G}) .
\end{array}\right.
$$

We recall that $S(t)$ is the random propagator associated with problem (5.5) such that $u(t)=S(t) f$. Our interest is again to prove a link between the convergence of $S(t)$ towards the orthogonal projector $P_{0}$ with the connectedness of the union of the graphs in $\mathrm{C}$. However, the key point here is to give a definition of the concept of union graph in the metric setting: this follows immediately from the above formalism, see [30].

Definition 5.4 (Union and intersection of metric graphs)

Let $\mathcal{G}_{1}, \ldots, \mathcal{G}_{N}$ be metric graphs defined on the same $\mathcal{E}$, i.e., $\mathcal{G}_{i}=\mathcal{E} / \equiv_{i}, i=$ $1, \ldots, N$. Denote by $\equiv \cup$ and by $\equiv \cap$ the equivalence relations obtained by taking the reflexive, symmetric, and transitive closure of $\bigcup_{i=1}^{N} \equiv_{i} \subset \mathcal{V} \times \mathcal{V}$ and $\bigcap_{i=1}^{N} \equiv_{i} \subset \mathcal{V} \times \mathcal{V}$, respectively. Then, we call union and intersection metric graph the metric graphs

$$
\mathcal{G} \cup:=\mathcal{E} / \equiv \cup \quad \text { and } \quad \mathcal{G}_{\cap}:=\mathcal{E} / \equiv \cap \text {, }
$$

respectively.

In Fig. 3 we can consider some examples of union graphs. 

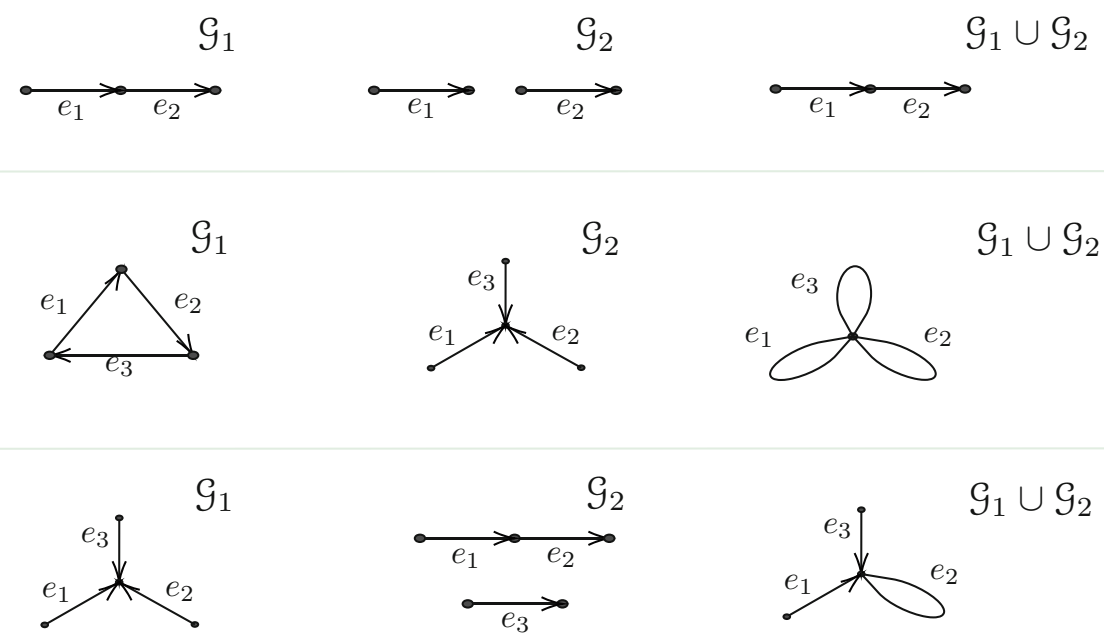

Fig. 3 Some examples of union graph
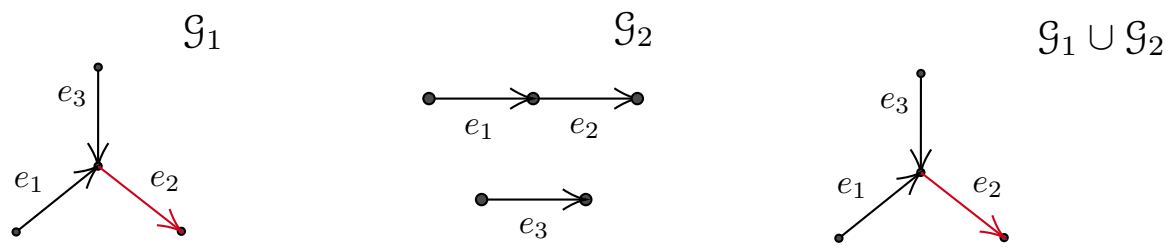

Fig. 4 If we reverse the orientation of just one edge, the resulting union is different

Remark 5.5 We observe that for fixed $\equiv_{1}, \equiv_{2}$, the union metric graph $\mathcal{G}_{1} \cup \mathcal{G}_{2}$ does depend on the orientations of the edges in $\mathcal{E}$ (as so do $\mathcal{G}_{1}, \mathcal{G}_{2}$, too); this is in sharp contrast to the case of combinatorial graphs.

For instance, we can take the same graphs $\mathcal{G}_{1}$ and $\mathcal{G}_{2}$ in the third example in Fig. 3 and just reverse the orientation of one edge as shown in Fig. 4.

Our main result in this section is the following lemma, which characterizes the null space of elliptic operators with natural vertex conditions associated with the union graph with its connectedness.

Lemma 5.6 Given $\mathcal{G}_{1}, \ldots, \mathcal{G}_{N}$ metric graphs satisfying the Assumption 5.3, let $\mathcal{G}$ be their union graph (see Definition 5.4). Let $A_{i}$ be the elliptic operators associated with $\mathcal{G}_{i}$ with natural vertex conditions operators and coefficients $p_{i} \in W^{1, \infty}\left(\mathcal{G}_{i}\right)$. Then

$$
\mathcal{G} \text { is connected } \Longleftrightarrow \bigcap_{i=1}^{N} \operatorname{ker} A_{i}=\langle\mathbb{1}\rangle \text {. }
$$

Notice that this lemma is remarkably similar to Corollary 4.5 (which deals with combinatorial graphs) and also their proofs will be similar. 
Proof We show the proof for $N=2$, then one can easily extend the result for an arbitrary $N$ by induction. In general, both $\mathcal{G}_{1}$ and $\mathcal{G}_{2}$ have a certain number of disjoint connected components:

$$
\mathcal{G}_{1}^{(1)}, \ldots, \mathcal{G}_{1}^{(m)} \text { s.t. } \mathcal{G}_{1}=\bigsqcup_{j=1}^{m} \mathcal{G}_{1}^{(j)}, \quad \text { for some } m \in \mathbb{N}
$$

and

$$
\mathcal{G}_{2}^{(1)}, \ldots, \mathcal{G}_{2}^{(n)} \text { s.t. } \mathcal{G}_{2}=\bigsqcup_{k=1}^{n} \mathcal{G}_{2}^{(k)}, \quad \text { for some } n \in \mathbb{N} \text {. }
$$

Since connectedness is just a topological property, notice that the connected components remain the same for every choice of orientation.

Now assume that $\mathcal{G}$ is connected: we need to show that $\operatorname{ker} A_{1} \cap \operatorname{ker} A_{2} \subseteq\langle\mathbb{1}\rangle$. Thus, we take $f \in \operatorname{ker} A_{1} \cap \operatorname{ker} A_{2}$, in particular from the results in Proposition 5.2 it is well-known that $f$ is constant on each connected component of both $\mathcal{G}_{1}$ and $\mathcal{G}_{2}$. Take $\xi=\left(x, \mathrm{e}_{h}\right)$ and $\theta=\left(y, \mathrm{e}_{l}\right)$ in $\mathcal{G}$ : we are going to show that

$$
f(\xi)=f(\theta) .
$$

(We can assume that $h \neq l$, otherwise the assertion is trivial.) By connectedness of $\mathcal{G}$, there exists a chain of adjacent (in $\mathcal{G}_{1}$ and/or in $\mathcal{G}_{2}$ ) edges $\Gamma \xi \theta=\left\{\mathrm{e}_{h}, \mathrm{e}_{i_{1}}, \ldots, \mathrm{e}_{i_{M}}, \mathrm{e}_{l}\right\}$ linking $\xi$ and $\theta$ :

$$
\xi \in \mathrm{e}_{h} \sim \mathrm{e}_{i_{1}} \sim \ldots \sim \mathrm{e}_{i_{M}} \sim \mathrm{e}_{l} \ni \theta
$$

Thus, taking into account that $f$ is constant on the connected components of both graphs, we deduce that $f$ is constant along $\Gamma_{x y}$ and in particular $f(\xi)=f(\theta)$. Because $\xi, \theta$ are arbitrary, we conclude that $f$ is constant.

In order to prove the opposite implication, we are going to show that if $\mathcal{G}$ is disconnected, then we can find a non constant function such that $f \in \operatorname{ker} A_{1} \cap \operatorname{ker} A_{2}$. Take two connected components $\mathcal{G}^{(A)}$ and $\mathcal{G}^{(B)}$ of $\mathcal{G}$. Then, both contain a certain number of connected components of $\mathcal{G}_{1}$ and $\mathcal{G}_{2}$. In particular, we set

$$
J_{A}=\left\{j \in\{1, \ldots, m\}: \mathcal{G}_{1}^{(j)} \subseteq \mathcal{G}^{(A)}\right\}, \quad J_{B}=\left\{j \in\{1, \ldots, m\}: \mathcal{G}_{1}^{(j)} \subseteq \mathcal{G}^{(B)}\right\}
$$

and

$$
K_{A}=\left\{k \in\{1, \ldots, n\}: \mathcal{G}_{2}^{(k)} \subseteq \mathcal{G}^{(A)}\right\}, \quad K_{B}=\left\{k \in\{1, \ldots, n\}: \mathcal{G}_{2}^{(k)} \subseteq \mathcal{G}^{(B)}\right\} .
$$

Due to the fact that $\mathcal{G}$ is disconnected, it follows that

$$
J_{A} \cap J_{B}=\emptyset, \quad K_{A} \cap K_{B}=\emptyset,
$$


in fact there exist no connected components, either of $\mathcal{G}_{1}$ or $\mathcal{G}_{2}$, shared by $\mathcal{G}^{(A)}$ and $\mathcal{G}^{(B)}$ : this is true regardless of the chosen orientation of the edges of $\mathcal{G}$. Hence, the indicator functions on each connected component of $\mathcal{G}_{1}$ (resp. $\mathcal{G}_{2}$ ) form a basis for the respective kernel

$$
\operatorname{ker} A_{1}=\left\langle\left\{\mathbb{1}_{1, j}\right\}_{j=1}^{m}\right\rangle \text { and } \operatorname{ker} A_{2}=\left\langle\left\{\mathbb{1}_{2, k}\right\}_{k=1}^{n}\right\rangle \text {. }
$$

Since every graph has the same set of edges (with possibly different connections), and every edge in $\mathcal{G}^{(A)}$ belongs to one and only one component $\mathcal{G}_{1}^{(j)}$ on $\mathcal{G}_{1}$ (and also to one and only one component $\mathcal{G}_{2}^{(k)}$ of $\mathcal{G}_{2}$ ) we can write

$$
\mathbb{1}_{A}=\sum_{\mathrm{e} \in \mathcal{G}^{(A)}} \mathbb{1}_{\mathrm{e}}=\left\{\begin{array}{l}
\sum_{j \in J_{A}} \sum_{\mathrm{e} \in \mathcal{G}_{1}^{(j)}} \mathbb{1}_{\mathrm{e}}=\sum_{j \in J_{A}} \mathbb{1}_{1, j} \\
\sum_{k \in K_{A}} \sum_{\mathrm{e} \in \mathcal{G}_{2}^{(k)}} \mathbb{1}_{\mathrm{e}}=\sum_{k \in K_{A}} \mathbb{1}_{2, j}
\end{array}\right.
$$

and similarly for $\mathcal{G}^{(B)}$ :

$$
\mathbb{1}_{B}=\sum_{\mathrm{e} \in \mathcal{G}^{(B)}} \mathbb{1}_{\mathrm{e}}=\left\{\begin{array}{l}
\sum_{j \in J_{B}} \sum_{\mathrm{e} \in \mathcal{G}_{1}^{(j)}} \mathbb{1}_{\mathrm{e}}=\sum_{j \in J_{B}} \mathbb{1}_{1, j} \\
\sum_{k \in K_{B}} \sum_{\mathrm{e} \in \mathcal{G}_{2}^{(k)}} \mathbb{1}_{\mathrm{e}}=\sum_{k \in K_{B}} \mathbb{1}_{2, j} .
\end{array}\right.
$$

At this point, we only need to take any function of the form

$$
f=\alpha \mathbb{1}_{A}+\beta \mathbb{1}_{B}, \quad \alpha, \beta \in \mathbb{C}, \alpha \neq \beta,
$$

and from (5.7) and (5.8) one gets that $f$ can be written as a linear combination of elements of the bases of both $\operatorname{ker} A_{1}$ and $\operatorname{ker} A_{2}$ :

$$
f=\alpha \sum_{j \in J_{A}} \mathbb{1}_{1, j}+\beta \sum_{j \in J_{B}} \mathbb{1}_{1, j} \quad \Longrightarrow \quad f \in \operatorname{ker} A_{1}
$$

and

$$
f=\alpha \sum_{k \in K_{A}} \mathbb{1}_{2, k}+\beta \sum_{k \in K_{B}} \mathbb{1}_{2, k} \quad \Longrightarrow \quad f \in \operatorname{ker} A_{2}
$$

Thus, the proof is complete.

In the end, we can finally state the following characterization of the asymptotic behavior of the solutions to (5.5) in terms of the connectedness of the union graph. The proof is, at this point, a direct consequence of Theorem 2.11 and Lemma 5.6. 
Theorem 5.7 Let $(Z(t))_{t \geq 0}$ be a semi-Markov process and $\mathcal{C}$ be a family of graphs that satisfy the Assumptions 2.1 and 5.3, respectively. Then the random propagator $(S(t))_{t \geq 0}$ for the Cauchy problem (5.5) converges in norm $\mathbb{P}$-almost surely towards the orthogonal projector $P_{0}$ onto the constants if and only if the union graph $\mathcal{G}$ is connected.

\subsection{Randomly Switching Metric Graphs with Non-zero Second Eigenvalue}

As we have previously seen in the combinatorial setting, we are going to apply exponential convergence results in the framework of metric graphs. We shall work under the following additional assumption.

Assumption 5.8 There exists one metric graph in $\mathcal{C}$, say $\mathcal{G}_{1}$, such that each connected component of $\mathcal{G}_{j}, j \neq 1$, is contained in one of the connected components of $\mathcal{G}_{1}$.

By a similar argument as in the proof of Lemma 5.6, this implies that the null space ker $A_{1}$ is contained in all the null spaces of $A_{j}, j \neq 1$, thus Assumption 2.12 is verified. The application of Theorem 2.13 then reads as follows.

Corollary 5.9 Let $(Z(t))_{t \geq 0}$ be a semi-Markov process and $\mathrm{C}$ be a family of graphs that satisfy the Assumptions 2.1 and 5.3, respectively. Let additionally the Assumption 5.8 hold.

Then the random propagator $(S(t))_{t \geq 0}$ for the Cauchy problem (5.5) converges in norm $\mathbb{P}$-almost surely exponentially fast towards the orthogonal projector $P_{K}$ with an exponential rate no lower than

$$
\alpha=-\sum_{j=1}^{N} s_{d}\left(A_{j}\right) \Theta_{j}
$$

that is the average of the values $s_{d}\left(A_{j}\right)$ (introduced in Remark 2.9) with respect to the fraction of time $\Theta_{j}$ spent by the process $Z(t)$ in the various states.

The setting described here is somehow comparable to the diffusion equation presented in [2], in the case when their semilinear term is set equal to zero. The dependence on time of that model is different from the non-autonomous random evolution problem (5.5): while diffusion and conductivity coefficients are in [2] allowed to vary over time (in a measurable fashion), yielding an operator family $(A(t))_{t \geq 0}$, the evolution is studied on one fixed graph. However-much like in our setting - the crucial point in [2] is that the time average of the spectral gap of $(A(t))_{t \geq 0}$ is bounded above away from zero. In their case, this is enforced by assuming that the graph is connected and allows the authors of [2] to prove exponential convergence to equilibrium.

Remark 5.10 As in the case of combinatorial graphs discussed in Remark 4.12, we can find in the literature some estimate on the best possible value of the parameter $\alpha$, provided that $\mathcal{G}_{1}$ is connected (in this case, Assumption 5.8 is satisfied and $s_{d}\left(A_{1}\right)=$ $\left.\lambda_{1}\left(A_{1}\right)\right)$. We refer e.g. to the estimates in [32, Théo. 3.1], [21, Thm. 1], and [24, Thm. 4.2]: for a generic connected metric graph $\mathcal{G}$ 


$$
-\frac{\pi^{2}|\mathrm{E}|^{2}}{L^{2}} \leq \lambda_{2} \leq-\frac{\pi^{2}}{L^{2}},
$$

where the second inequality is an equality if and only if $\mathcal{G}$ consists of an interval; here $|\mathrm{E}|$ is the number of edges and $L$ is the total length of the graph (the sum of the lengths of the edges). Therefore, the parameter $\alpha$, that is the weighted average of $-\lambda_{2}\left(A_{i}\right)$ as $\mathcal{G}_{i}$ varies in $\mathcal{C}$, is no lower than $\frac{\pi^{2}}{L^{2}}$ (as long as the intersection graph $\mathcal{G} \cap$ of all graphs in $\mathcal{C}$ is connected) and no higher than $\frac{\pi^{2}|\mathrm{E}|^{2}}{L^{2}}$.

Acknowledgements The authors would like to thank Jochen Glück (Passau) and Marvin Plümer (Hagen) for their help in the proof of Lemma 3.2; Valter Moretti (Trento) for several useful discussions concerning Lemma 3.3 and the anonymous referees for careful reading and useful comments.

Funding Open access funding provided by Universitá degli Studi di Trento within the CRUI-CARE Agreement.

Open Access This article is licensed under a Creative Commons Attribution 4.0 International License, which permits use, sharing, adaptation, distribution and reproduction in any medium or format, as long as you give appropriate credit to the original author(s) and the source, provide a link to the Creative Commons licence, and indicate if changes were made. The images or other third party material in this article are included in the article's Creative Commons licence, unless indicated otherwise in a credit line to the material. If material is not included in the article's Creative Commons licence and your intended use is not permitted by statutory regulation or exceeds the permitted use, you will need to obtain permission directly from the copyright holder. To view a copy of this licence, visit http://creativecommons.org/licenses/by/4.0/.

\section{References}

1. Acosta, María D.: Denseness of norm attaining mappings. RACSAM 100(1-2), 9-30 (2006)

2. Arendt, W., Dier, D., Kramar Fijavž, M.: Diffusion in networks with time-dependent transmission conditions. Appl. Math. Optim. 69, 315-336 (2014)

3. Acquistapace, P., Terreni, B.: A unified approach to abstract linear nonautonomous parabolic equations. Rendiconti del seminario matematico della Università di Padova 78, 47-107 (1987)

4. Burago, D., Burago, Y., Ivanov, S.: A Course in Metric Geometry. Graduate Studies in Mathematics, vol. 33. American Mathematical Society, Providence, RI (2001)

5. Bakhtin, Yuri, Hurth, Tobias: Invariant densities for dynamical systems with random switching. Nonlinearity 25(10), 2937-2952 (2012)

6. Berkolaiko, G., Kuchment, P.: Introduction to Quantum Graphs. Mathematical Surveys and Monographs, vol. 186. American Mathematical Society, Providence, RI (2013)

7. Benaïm, M., Le Borgne, S., Malrieu, F., Zitt, P.-A.: Quantitative ergodicity for some switched dynamical systems. Electron. Commun. Probab. 17(56), 14 (2012)

8. Benaïm, M., Le Borgne, S., Malrieu, F., Zitt, P.-A.: On the stability of planar randomly switched systems. Ann. Appl. Probab. 24(1), 292-311 (2014)

9. Buceta, J., Lindenberg, Katja., Parrondo, J. M. R.: Global alternation-induced patterns. Fluct. Noise Lett., 2(4):R139-R159, (2002). Game theory and evolutionary processes: order from disorder-the role of noise in creative processes

10. Bressloff, P.C.: Stochastic switching in biology: from genotype to phenotype. J. Phys. A 50, 136 (2017)

11. Cloez, B., Hairer, M.: Exponential ergodicity for Markov processes with random switching. Bernoulli 21(1), 505-536 (2015)

12. Davis, M.H.A.: Piecewise-deterministic Markov processes: a general class of non-diffusion stochastic models. J. R. Stat. Soc. Series B 46, 353-376 (1984) 
13. Daners, D., Glück, J., Kennedy, J.B.: Eventually positive semigroups of linear operators. J. Math. Anal. Appl. 433, 1561-1593 (2016)

14. Daubechies, I., Lagarias, J.C.: Sets of matrices all infinite products of which converge. Linear Algebra Appl. 161, 227-263 (1992)

15. Dautray, R., Lions, J.-L.: Mathematical Analysis and Numerical Methods for Science and Technology, vol. 5. Springer, Berlin (1992)

16. Daubechies, I., Lagarias, J.C.: Corrigendum/addendum to: sets of matrices all infinite products of which converge. Linear Algebra Appl. 327, 69-83 (2001)

17. Elsner, L., Koltracht, I., Neumann, M.: On the convergence of asynchronous paracontractions with application to tomographic reconstruction from incomplete data. Linear Algebra Appl. 130, 65-82 (1990)

18. Engel, K.-J., Nagel, R.: One-Parameter Semigroups for Linear Evolution Equations. Graduate Texts in Mathematics, vol. 194. Springer, New York (2000)

19. Fiedler, M.: Algebraic connectivity of graphs. Czech. Math. J. 23, 298-305 (1973)

20. Furstenberg, H., Kesten, H.: Products of random matrices. Ann. Math. Stat. 31, 457-469 (1960)

21. Friedlander, L.: Extremal properties of eigenvalues for a metric graph. Ann. Inst. Fourier 55, 199-212 (2005)

22. Gurvits, Leonid: Stability of discrete linear inclusion. Linear Algebra Appl. 231, 47-85 (1995)

23. Hussein, A., Mugnolo, D.: If time were a graph, what would evolution equations look like? arXiv:2001.06868

24. Kennedy, J.B., Kurasov, P., Malenová, G., Mugnolo, D.: On the spectral gap of a quantum graph. Ann. Henri Poincaré 17, 2439-2473 (2016)

25. Kramar Fijavž, M., Mugnolo, D., Sikolya, E.: Variational and semigroup methods for waves and diffusion in networks. Appl. Math. Optim. 55, 219-240 (2007)

26. Korolyuk, V., Swishchuk, A.: Semi-Markov random evolutions, volume 308 of Mathematics and its Applications. Kluwer Academic Publishers, Dordrecht, 1995. Translated from the (1992 )Russian original by V. Zayats and revised by the authors

27. Lawley, S.D.: Blowup from randomly switching between stable boundary conditions for the heat equation. Commun. Math. Sci. 16(4), 1133-1156 (2018)

28. Levy, Paul.: Processus semi-markoviens. In: Proceedings of the International Congress of Mathematicians, 1954, Amsterdam, vol. III, pp. 416-426. Erven P. Noordhoff N.V., Groningen; North-Holland Publishing Co., Amsterdam (1956)

29. Malrieu, F.: Some simple but challenging Markov processes. Ann. Fac. Sci. Toulouse Math. 24(4), 857-883 (2015)

30. Mugnolo, D.: What is actually a metric graph? arXiv:1912.07549

31. Mugnolo, D.: Semigroup Methods for Evolution Equations on Networks. Complex Systems are Systems. Springer, Berlin (2014)

32. Nicaise, S.: Spectre des réseaux topologiques finis. Bull. Sci. Math., II. Sér. 111, 401-413 (1987)

33. Pyke, R., Schaufele, R.: Limit theorems for Markov renewal processes. Ann. Math. Stat. 35, 1746-1764 (1964)

34. Pyke, R.: Markov renewal processes with finitely many states. Ann. Math. Stat. 32, 1243-1259 (1961)

35. Schmüdgen, K.: Unbounded Self-adjoint Operators on Hilbert Space. Graduate Texts in Mathematics, vol. 265. Springer, Berlin (2012)

36. Smith, W.L.: Regenerative stochastic processes. Proc. R. Soc. Lond. Ser. A 232, 6-31 (1955)

37. Tanabe, H.: Equations of Evolution. Monographs and Studies in Mathematics, vol. 6. Pitman, Boston (1979)

38. George Yin, G., Zhu, Chao.: Hybrid switching diffusions, volume 63 of Stochastic Modelling and Applied Probability. Springer, New York, (2010). Properties and applications

39. Zeitouni, O.: Random walks in random environments. In: Proceeding of the International Congress of Mathematicians (Beijing 2002), volume 3, pp. 117-127, Beijing, (2002). Higher Ed. Press

40. Zeitouni, O.: Random walks in random environment. In: S. Tavaré and O. Zeitouni, editors, Lectures on Probability Theory and Statistics, volume 1837 of Lect. Notes Math., pp. 190-312. Springer, Berlin (2004)

Publisher's Note Springer Nature remains neutral with regard to jurisdictional claims in published maps and institutional affiliations. 